\title{
External temperature sensor assisted a new low power photoplethysmography readout system for accurate measurement of the bio-signs
}

\author{
Rajeev Kumar Pandey ${ }^{1} \cdot$ Paul C.-P. Chao ${ }^{2}$
}

Received: 1 November 2020 / Accepted: 7 November 2020/Published online: 27 November 2020

(C) Springer-Verlag GmbH Germany, part of Springer Nature 2020

\begin{abstract}
This study presents an external temperature sensor assisted a new low power, time-interleave, wide dynamic range, and low DC drift photoplethysmography (PPG) signal acquisition system to obtain the accurate measurement of various bio signs in real-time. The designed chip incorporates a 2-bit control programmable transimpedance amplifier (TIA), a high order filter, a 3:8 programmable gain amplifier (PGA) and $2 \times 2$ organic light-emitting diode (OLED) driver. Temperature sensor is used herein to compensate the adverse effect of low-skin-temperature on the PPG signal quality. The analog front-end circuit is implemented in the integrated chip with chip area of $2008 \mu \mathrm{m} \times 1377 \mu \mathrm{m}$ and fabricated via TSMC T18 process. With the standard $1.8 \mathrm{~V}$, the experimental result shows that the measured current sensing range is $20 \mathrm{nA}-100 \mathrm{uA}$. The measured dynamic range of the designed readout circuit is $80 \mathrm{~dB}$. The estimated signal to noise ratio is $60 \mathrm{~dB} @ 1 \mathrm{uA}$, and the measured input referred noise is $60.2 \mathrm{pA} / \mathrm{Hz}^{1 / 2}$. The total power consumption of the designed chip is $31.32 \mu \mathrm{W}$ (readout) + $1.62 \mathrm{~mW}$ (OLED driver@100\% duty cycle). The non-invasive PPG sensor is applied to the wrist artery of the 40 healthy subjects for sensing the pulsation of the blood vessel. The experimental results show that for every $1{ }^{\circ} \mathrm{C}$ decrease in mean ambient temperature tends to 0.06 beats $/ \mathrm{min}, 0.125 \mathrm{mmHg}$ and $0.063 \mathrm{mmHg}$ increase in hear rate (HR), systolic (SBP) and diastolic (DBP), respectively. Similarly, for every $1{ }^{\circ} \mathrm{C}$ increase in mean ambient temperature tends to 0.13 beats/min, $0.601 \mathrm{mmHg}$ and $0.121 \mathrm{mmHg}$ increase in HR, SBP and DBP, respectively. The measured accuracy and standard error for the HR estimation are $96 \%$, and $-0.022 \pm 2.589$ beats/minute, respectively. The oxygen stauration $\left(\mathrm{S}_{\mathrm{p}} \mathrm{O}_{2}\right)$ measurement results shows that the mean absolute percentage error is less than 5\%. The resultant errors for the SBP and DBP measurement are $-0.318 \pm 5.19 \mathrm{mmHg}$ and $-0.5 \pm 1.91 \mathrm{mmHg}$, respectively.
\end{abstract}

\section{Introduction}

With the rise of coronavirus disease (COVID-19) pandemic, vital bio signs like blood oxygen saturation $\left(\mathrm{S}_{\mathrm{p}} \mathrm{O}_{2}\right)$, body temperature, heart rate (HR) and blood pressure (BP) becomes one of the major concerns. According to the

Electronic supplementary material The online version of this article (https://doi.org/10.1007/s00542-020-05106-y) contains supplementary material, which is available to authorized users.

Paul C.-P. Chao

pchao@mail.nctu.edu.tw

1 EECS International Graduate Program, National Chiao Tung University, Hsinchu 300, Taiwan

2 Department of Electrical Engineering, National Chiao Tung University, Hsinchu 300, Taiwan
WHO report, about 17.9 million peoples die every year due to cardiovascular disease, that is $31 \%$ percent of all the global deaths. Heart rate variability (HRV) helps to estimate the physical condition of the body and it is highly important for the patient those who have the disease like atrial fibrillation. Higher value of the BP caused hypertension and it is measured in terms of the SBP and DBP. Low value of the $\mathrm{S}_{\mathrm{p}} \mathrm{O}_{2}$ causes hypoxia condition, that happens when the body does not get enough oxygen. Therefore, the monitoring of the all the aforementioned bio signs is necessary to track the health condition and eventually helps to reduce down the mortality rate. Point to be noted that the hospital-based devices are expensive and bulky, and their uses are only limited to hospitals and health clinics. To enable individuals interested in tracking their body's key health indicators, wearable devices are one of the main choices. In recent past, many state-of-arts were dedicated to develop a wearable sensor that can measure 
long-time, continuous and comfortable HR and BP. Most of them employed either electrocardiogram (ECG) and/or photoplethysmography (PPG) sensors (Ahmad et al. 2012; Forouzanfar et al. 2013; Huang et al. 2014, Cohen et al. 2017; Sommermeyer et al. 2016; Zheng et al. 2014; Tang et al. 2017; Sharma et al. 2017; Yan et al. 2019). The usage of the ECG devices is limited for the long-time-continuous monitoring of the BP-HR because the ECG device needs a skin preparation and close loop before the measurement. Also, the high skin impedance and the signal integrity adversely cause the base line wander, substantial noise (artifact) and even loss of the ECG trace (Bonomi et al. 2016). Unlike ECG, PPG devices are easy to wear and it can measure the light reflected from the wrist artery due to varying blood volume in the capillaries. PPG devices is not only used for sensing the $\mathrm{S}_{\mathrm{p}} \mathrm{O}_{2}$ and $\mathrm{HR}$, but also it can be used to estimate $\mathrm{BP}$. The long-time-continuous monitoring of the bio-sign using PPG devices is always a challenge because these devices are suffered from the mispositioning, low signal to noise ratio (SNR). In addition, PPG based devices are highly affected by the motion artifact and ambient noise artifact (Khan et al. 2019), temperature variation and the skin color (Khan et al. 2015; Pandey et al. 2019). Usually the AC/DC ratio associated with LED-PD PPG sensors are less than $10 \%$. Therefore, a slight motion or the ambient light induces a significant change in the DC component $(<0.1 \mathrm{~Hz})$ in such a way that the detection of the AC component due to pulsation becomes extremely difficult. Similarly, the high frequency noise $(>10 \mathrm{~Hz})$ reduces the SNR. On the other hand, with lowering the temperature, the AC/DC ratio degrade. Similarly, with high temperature the AC/DC ratio is increases significantly. Considering all the above mention challenges associated with the PPG sensors, in the recent past, many researchers have dedicated their effoert to developed a long time continuous and accurate measurement of the bio sign using the PPG devices (Wu et al. 2020; Marefat et al. 2020; Lin et al. 2019). Khan et al. (2019) proposed a flexible organic sensor for the health monitoring. A rectangular, bracket and circular geometry were design to achieve the improved the AC/DC. Sharma et al. (2017) proposed a sub $60 \mu \mathrm{A}$ multimodal PPG sensing circuit with the large SNR ( $>80 \mathrm{~dB})$. In this study adaptive sampling and adaptive intensity control are the main control mechanism to achieve the low power and high SNR. Marefat et al. (2020) proposed a $1 \mathrm{~V}, 8.1 \mu \mathrm{A}$ with $>92 \mathrm{db}$ dynamic range AFE for the PPG signal Acquisition. However, the performance of the designed circuit is validated only on the fingertip, and the minimum current sense range is still unknown. Similarly, Lin et al. (2019) proposed a reconfigurable dynamic range light to digital converter by using variable pulse repetition frequency (PRF). However, in terms of the low current sense the dynamic power consumption and the kick back noise from the comparator are a major concern. Similarly, Lee et al. (2017) proposed an OLED-OPD based sticker type hybrid system to compensate the effect of the motion artifact. However, the process of the ambient light rejection and large DC current rejection using the adpative filter control feedback current source are limited for the real time application. Thence an adaptive wide dynamic range (DR), high sensing precision to low current, high signal to noise ratio (SNR) are the main requirement of the PPG acquisition system.

Body temperature is the equilibrium temperature between the heat production and heat loss in the body. This is divided int two parts: skin temperature and core temperature. Madaniyazi et al. (2016), demonstrate the effect of the cold and hot temperature on the HR, SBP and DBP. According to this study, increase and decrease in the temperature beyond a limit will result in increase in HR, SBP and DBP. In addition, the value of the $\mathrm{S}_{\mathrm{p}} \mathrm{O}_{2}$ is also affected by the temperature. Higgins et al. (2014) demonstrate the effect of the temperature by using oxygen dissociation curve (ODC). Considering the environmental temperature effect, it is experimentally proven that at the low skin temperature $\left(<20^{\circ} \mathrm{C}\right) \mathrm{AC} / \mathrm{DC}$ ratio degrade (Khan et al. 2015). It happens because the reduced scattering coefficient $\left(\mu_{\mathrm{a}}\right.$ and $\left.\mu_{\mathrm{s}}{ }^{\prime}\right)$ changes with the temperature. The parameter $\mu_{\mathrm{s}}$ ' changes linearly with the temperature. However, the change in $\mu_{\mathrm{a}}$ is irreversible for the long time. Similarly, the light penetration depth $(\delta)$ in skin increased on cooling with considerable individual skin (Khalil et al. 2003). Similarly, with the high skin temperature the AC/DC ratio is increase significantly. Therefore, such kind of the uncertainty, limit the accuracy ability of the PPG sensor for the long-time continuous monitoring of the bio-signs. Pandey et al. (2019) proposed a strategy to compensate the temperature effect. This study uses the environment temperature to compensate the impact of the temperature. That proposed remedy is valid only for the certain closed environment.

In this study an external temperature sensor assisted, a new adaptive PPG readout chip with an integrated adaptive OLED driver is proposed to measure the PPG signal from the OLED-OPD sensor patch. The designed PPG sensing patch is integrated with the temperature sensor on the same flexible patch to compensate the impact of the temperature on the PPG signal quality, as well as it helps to measure the continuous body temperature. The readout circuit with the OLED driver are implemented in the integrated chip with chip area of $2008 \times 1377 \mu \mathrm{m}^{2}$ and fabricated via TSMC T18 process. Experimental result shows that the achieved dynamic range of the AFE $>80 \mathrm{~dB}$. The design AFE can sense the 20nA of the minimum current. The total power consumption of the design chip is $31.32 \mu \mathrm{W}$ (readout $+1.62 \mathrm{~mW}$ (OLED@100\% duty cycle). Pre-signal 
processing, $\mathrm{S}_{\mathrm{p}} \mathrm{O}_{2}, \mathrm{HR}$ and $\mathrm{BP}$ estimation algorithm has been designed by using the MATLAB. The measured accuracy and standard error for the HR estimation are $96 \%$, and $-0.022 \pm 2.589 \mathrm{bpm}$, respectively. The $\mathrm{S}_{\mathrm{p}} \mathrm{O}_{2}$ measurement results shows that the means absolute percentage error is less than 5\%. The resultant errors for the SBP and DBP measurement are $-0.29 \pm 5.19 \mathrm{mmHg}$ and $-0.5 \pm 1.91 \mathrm{mmHg}$, respectively. The rest of the study is organized as follow: Section II presents the design of PPG Signal Acquisition System. Section III will discuss about the pre-signal processing, $\mathrm{HR}, \mathrm{S}_{\mathrm{p}} \mathrm{O}_{2}$ and $\mathrm{BP}$ estimation algorithm. Section IV presents the experimental results and finally section $\mathrm{V}$ concludes this study.

\section{Design of PPG signal acquisition sensor and system}

The block diagram of the PPG acquisition system is shown in Fig. 1, which incorporates an OLED-OPD PPG sensor, an adaptive readout circuit, an OLED driver circuit, a temperature sensor, a micro controller unit (MCU) and Bluetooth transceiver. Analog readout circuit incorporates the programmable transimpedance amplifier (PTIA), 2nd order high pass filter (HPF) with the cut off of $0.1 \mathrm{~Hz}, 4$ th order $\mathrm{Gm}-\mathrm{C}$ low pass filter (LPF) with a cut-off of $10 \mathrm{~Hz}$, and a programmable gain amplifier (PGA). The OLED driver incorporates the voltage control oscillator (VCO), Analog multiplexer (4:1), and a 1-bit DAC. Similarly, the internal part of the temperature sensor incorporate temperature senor $(\mathrm{BJT})$, readout and $\mathrm{I}^{2} \mathrm{C}$ interface. MATLAB

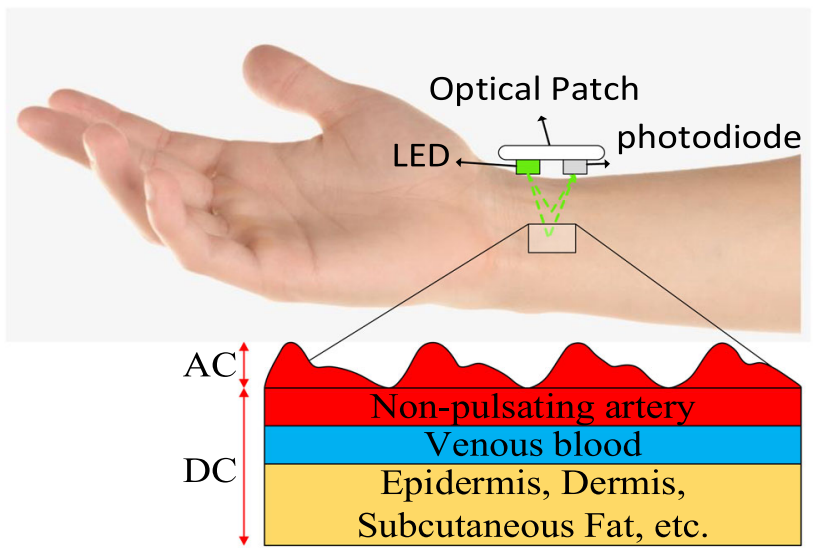

Fig. 2 Principle of the PPG signal using a photodetector and a LED (Pribadi et al. 2020)

has been used to designed the background software, which incorporates the pre-signal processing (FIR filtering, readout control algorithm), $\mathrm{S}_{\mathrm{p}} \mathrm{O}_{2}$, HR and $\mathrm{BP}$ estimation algorithm. The supply voltage of the whole circuit is $1.8 \mathrm{~V}$. The next sub-section will described the design of each sub block in details.

\subsection{Principle of the reflectance type PPG sensing}

PPG measures the changes in the intensity of light reflected by the user's skin due to varying volume and oxygenation of blood in the capillaries. Figure 2 shows the reflectance type PPG sensing to extract the PPG signals from the wrist artery. OLED emits the lights which penetrate the skin through the blood vessel, and then the light reflects back to

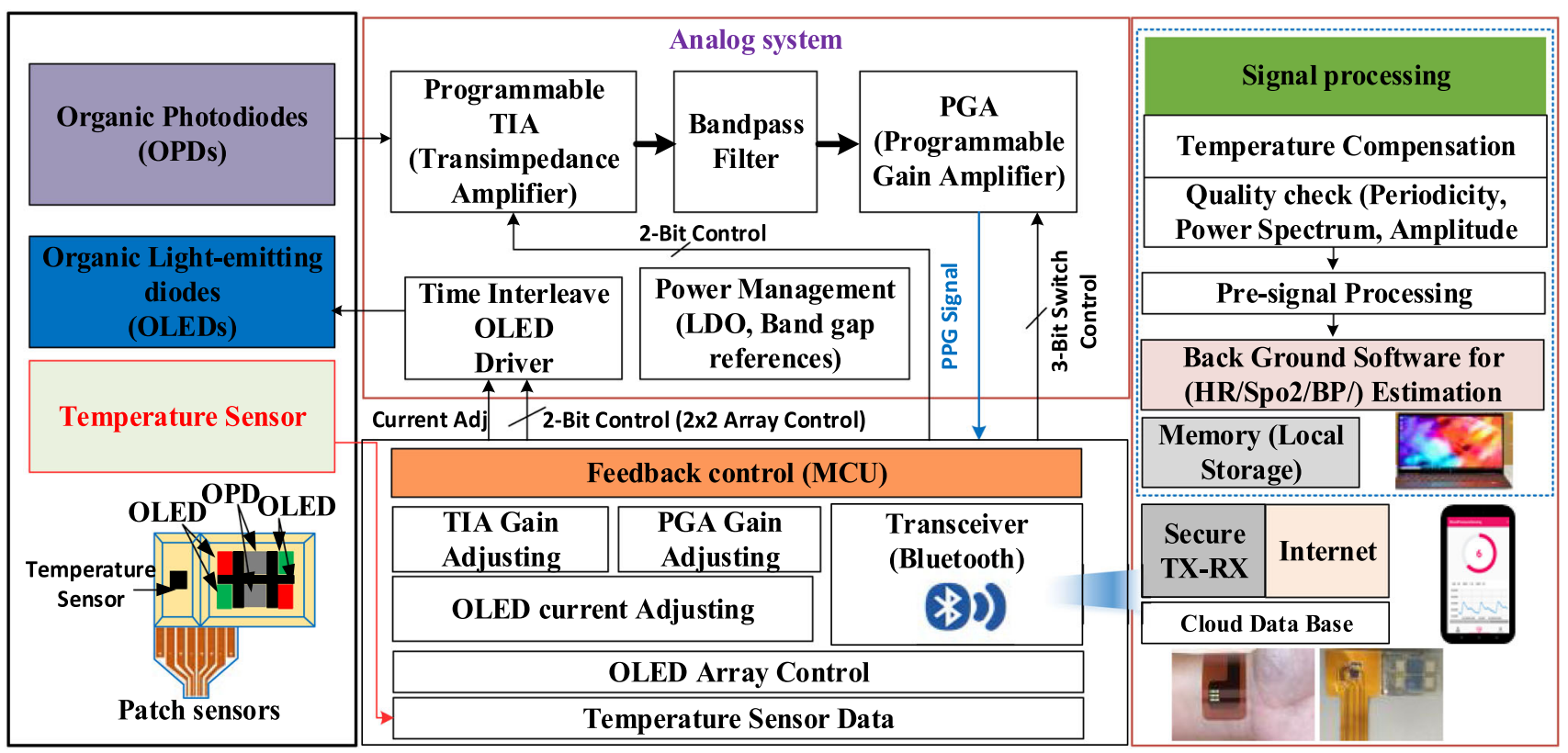

Fig. 1 Block Diagram of the temperature compensated PPG Readout System 


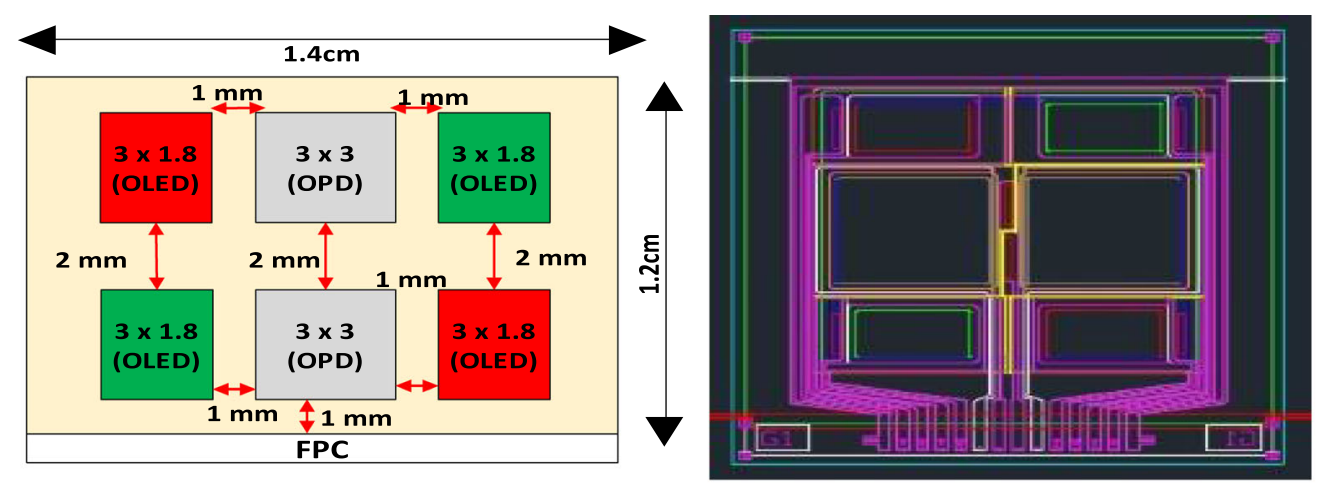

(a)
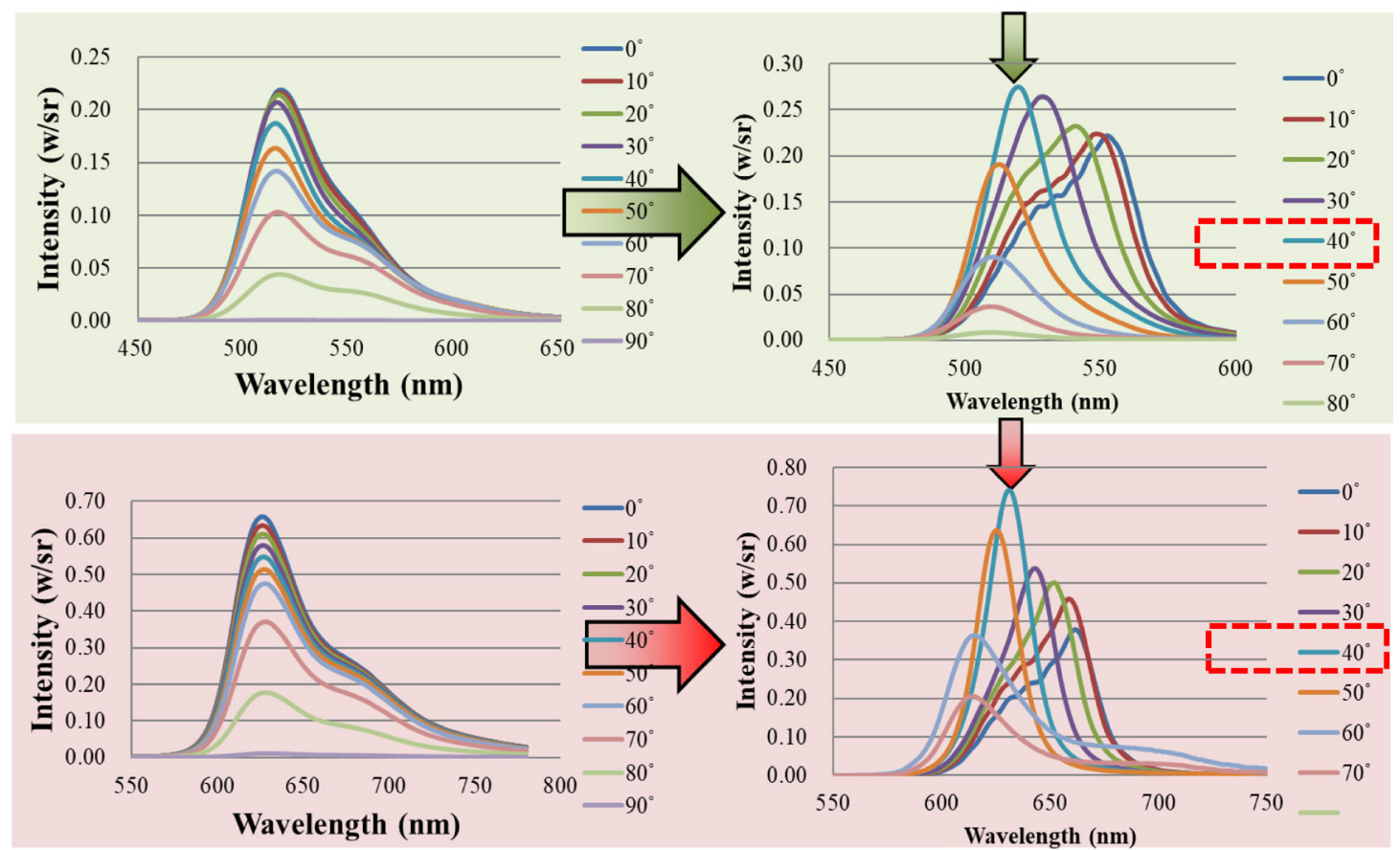

(b)

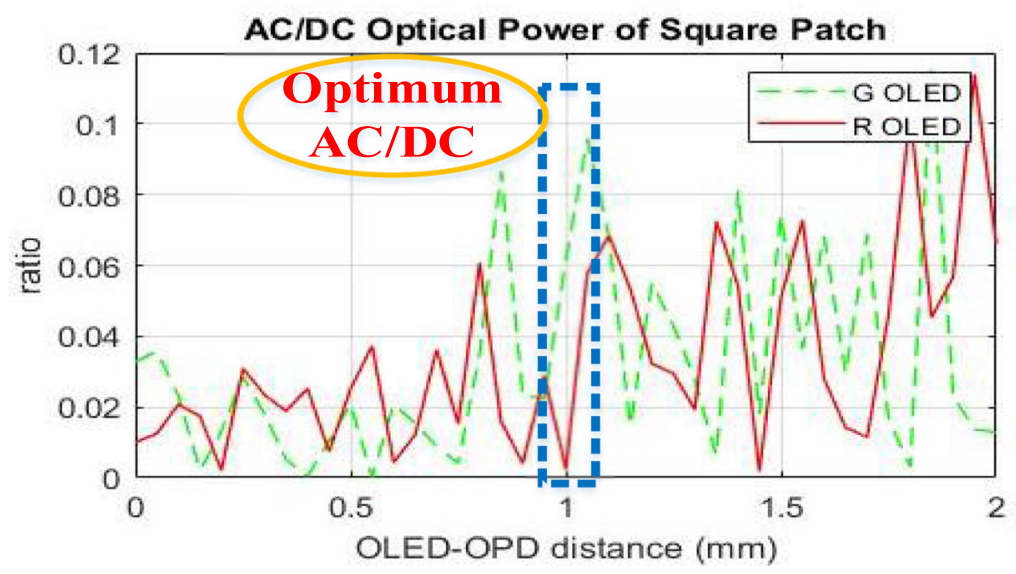

(c) 
4Fig. 3 a Physical view and layout of the rectangular type OLED-OPD sensor, $\mathbf{b}$ incidence angle optimization, $\mathbf{c}$ AC/DC ratio optimization (OLED-OPD distance optimization)

the OPD. The electric signal generated by PD is processed through the readout system to obtain a high-quality raw PPG signal.

\subsection{OLED-OPD sensor array design}

In this study a new reflective OLED-OPD flexible PPG array sensor is design and developed to minimize the issue of motion artifacts and mispositioning. The design of the OLED-OPD is shown in Fig. 2. It is extension of the previous study published in Pribadi' et al (2020). It consists of two red, two green OLED with $630 \mathrm{~nm}$ and $530 \mathrm{~nm}$ wavelength respectively. It also incorporates the two OPD. Optical simulation has been performed to optimize the aperture size of the OLED-OPD patch. For the simulation the tissue modelled by assuming the standard thicknesses of different layers under the skin, such as $0.1 \mathrm{~mm}$ to $0.14 \mathrm{~mm}$ for epidermis, $0.5 \mathrm{~mm}$ to $3 \mathrm{~mm}$ for dermis, and $3 \mathrm{~mm}$ to $50 \mathrm{~mm}$ for sub-cutis. The thickness of pulsating wrist artery is assumed as 2-2.2 mm (Pribadi et al. 2020; Pandey et al. 2019; Kao et al. 2019). Typically, the optical properties of these layers are characterized by absorption $\mu_{\mathrm{a}}$ and scattering $\mu_{\mathrm{s}}$ coefficients and the anisotropy factor $\mathrm{g}$. The absorption coefficient characterizes the average number of absorption events per unit path length of photons travelling in the tissue. The main absorbers in the visible spectral range are the blood, hemoglobin, $\beta$-carotene, and bilirubin. For the optical simulation, the skin model includes the reflection coefficient and scattering coefficient at $0{ }^{\circ} \mathrm{C}$ and $27^{\circ} \mathrm{C}$ temperature respectively. In order to obtain the maximum SNR in terms of AC/DC ratio, the distances between OLED \& OPD is varied from 0.6 to $2.5 \mathrm{~mm}$ with an increment of $0.1 \mathrm{~mm}$. The obtain optimized distance between the OLED-OPD is $1 \mathrm{~mm}$. The basic glass substrate element does not have a proper optical design consideration, and the brightness of observation under each viewing angle gradually declines. Therefore, under optical simulation the different viewing has been simulated and it is observed that at $40^{\circ}$ tilt angle, the observe intensity from the OLED is the highest. After verifying the view angle, OLED are fabricated. The verification results related to the tilt angle for Green and Red OLED are shown in the Fig. 3a. Figure $3 b$ shows the total $\mathrm{AC}$ pulsating power received in term of AC-to-DC ratio and it can be seen that the optimized distance between OLED-OPD is $1 \mathrm{~mm}$ and the received AC/DC ratio with green and red OLED are $7 \%$ and $8 \%$ respectively. The estimated OPD current is varied in the range of $50 \mathrm{nA}$ to the $11 \mathrm{uA}$ (including dark current). The rated OLED current is in the range of the $0.01-1.6 \mathrm{~mA}$ with the forward bias voltage varying from 0.1 to $3.3 \mathrm{~V}$.

\subsection{Programmable gain transimpedance amplifier}

The programmable low pass transimpedance amplifier (TIA) is used herein to convert the received photo diode current into an equivalent voltage. The OPD is operating in photovoltaic mode and the TIA keeps the bias voltage near REF while converting OPD current into voltage. Programmable gain TIA can amplify output voltage under low photocurrent from the OPD and also can keep the output in the linear region under high photocurrent from the OPD (Orozco 2013). The circuit diagram of the programmable gain transimpedance amplifier is shown in Fig. 4a. The designed TIA incorporate four-passive resistance, a fully differential operational transconductance amplifier and the switch control 2:4 decoder. The control signal from the microcontroller unit (MCU) is used to programmed the gain of the TIA. The internal circuit diagram of the fully-differential operational transconductance amplimer (OTA) is shown in Fig. 4b. The $-3-d B$ cut off frequency of the the designed TIA is varied from the 0.7 to $2.5 \mathrm{MHz}$, and it depend on the effective value of the feedback Resistance $\left(\mathrm{R}_{\text {Total }}\right)$. The overall transfer function of the low pass programmable TIA is given by

$\operatorname{Gain}_{\text {Loop } 1}(s)=\frac{\operatorname{Vout}(s)_{1 \text { ststage }}}{I_{O P D}}=\frac{A_{O P A 1}}{1+A_{O P A 1}} \frac{R_{\text {Total }}}{1+s R_{\text {Rotal }} C_{f}}$,

where $A_{O P A 1}$ is the gain of the OTA and $I_{O P D}$ is the sense current from the OPD. The frequency response of the TIA with $\mathrm{R}_{\text {Total }}=4 \mathrm{R} @$ five process corners is shown in Fig. 4c. The simulated maximum gain of the TIA is $110 \mathrm{~dB}(\mathrm{Ohm})$. The designed TIA can amplify the output voltage under low photocurrent from the OPD and also can keep the output in the linear region under high photocurrent from the OPD. Since the noise is a major concern so the inverting low pass unity gain amplifier (cut off frequency $<10 \mathrm{~Hz}, \quad \mathrm{C}_{2}=0.1 \mu \mathrm{F} \quad$ external capacitor, and $\mathrm{R}_{2}=220 \mathrm{~K}$ on chip) is used at the output of the TIA to minimize the impact of the high frequency noise, ambient noise and powerline noise. Point to be noted that the programmable TIA incorporates a 2:4 decoder with the control signal named as PC0 and PC1. Micro controller (MCU) compare the received PPG signal with min $(0.1 \mathrm{~V})$ and $\max (1.7 \mathrm{~V})$ threshold value and then automictically program the TIA effective feedback resistance by using the 2-bit control signal (PC0 and PC1). 


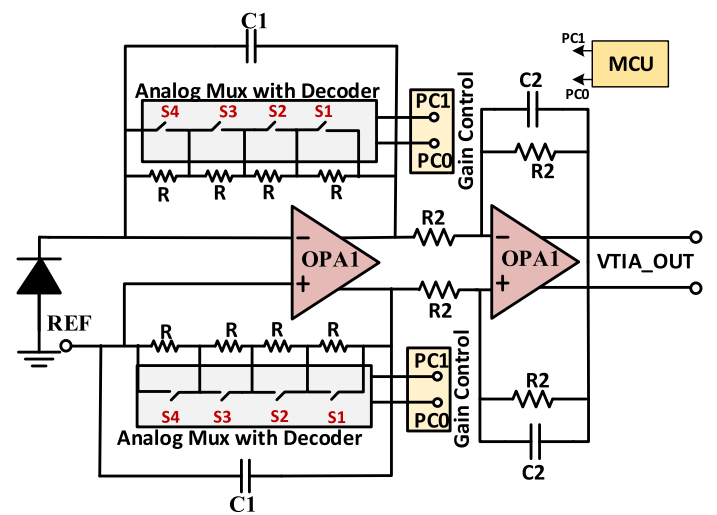

(a)

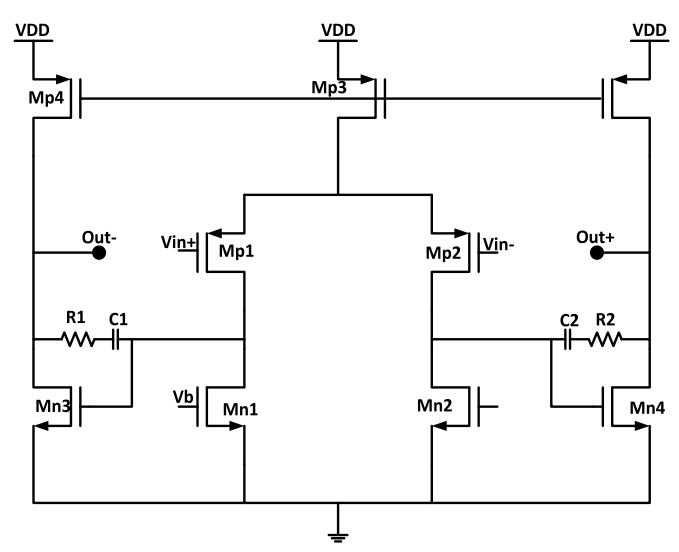

(b)

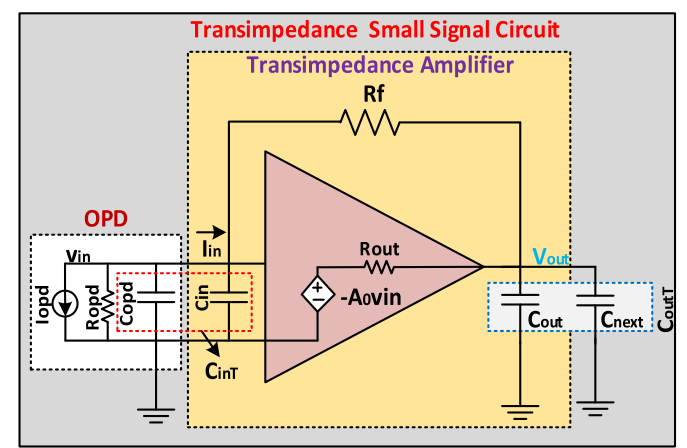

(c)

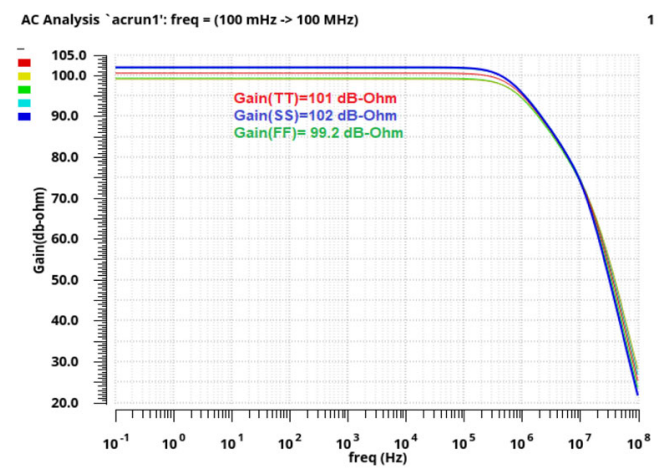

(d)
4Fig. 4 a Wide dynamic range programmable gain TIA, b circuit diagram of the OTA, c small signal model, $\mathbf{d}$ The frequency response of the TIA@4R with five process corners

\subsection{Second order high pass filter}

To remove the DC drift either because of the skin absorption and/or ambient and/or motion artifacts. A second-order high-pass filter circuit is used herein to reject the noise $<0.1 \mathrm{~Hz}$. The circuit diagram of the $1^{\text {st }}$ order high pass circuit is shown in the Fig. 5. The transfer function of high-pass filter is given by

$\frac{V o^{+}-V o^{-}}{V i^{+}-V i^{-}}=\frac{C_{1}}{C_{2}} \cdot \frac{1-\frac{s C_{2}}{G m}}{\left(\frac{1}{s R_{2} C_{2}+1}\right)\left(s \frac{C_{L} C_{1}}{G m C_{2}}+1\right)}$.

The ratio of the capacitance $C_{1}$ and $C_{2}$, determine the mid-band gain of the filter. The resistance R1 and the capacitance $C_{2}$ determine the high pass cut off as low as $0.1 \mathrm{~Hz}$. The output of the TIA is capacitively coupled through $C_{1}$ and therefore any dc offset from the input is removed. However, the value of the $C_{l}$ need to be smaller to minimize the signal attenuation. In order to achieve the cut off of $0.1 \mathrm{~Hz}$, the required value of the resistance is $1 \mathrm{G}$ $\mathrm{ohm}$. The controlled pseudo MOSFET resistance is used herein to realize the high value resistance (Guglielmi et al. 2020; Shiue et al. 2011). The circuit diagram of the pseudo resistance used in this study is shown in Fig. 6. Point to be

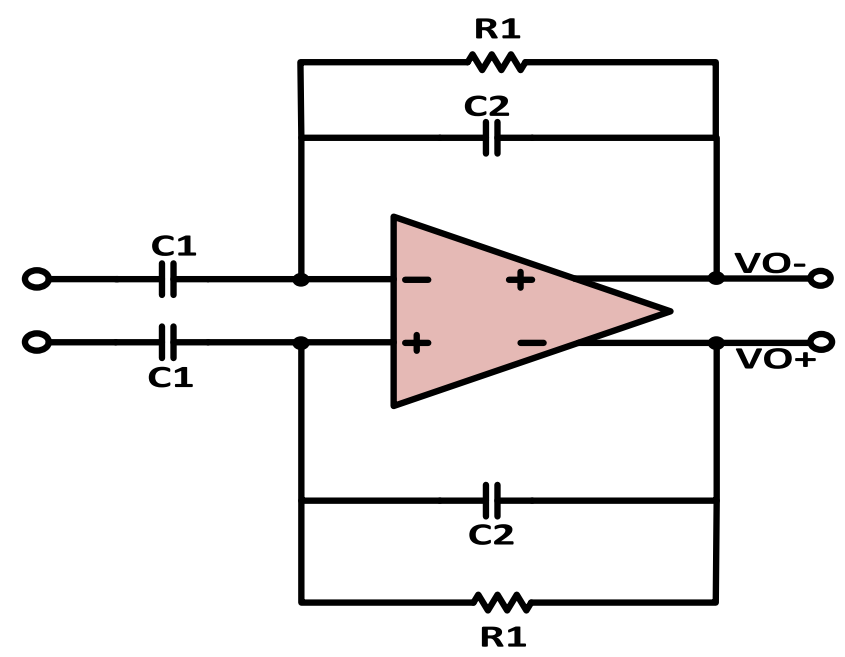

Fig. 5 First order high-pass filter circuit

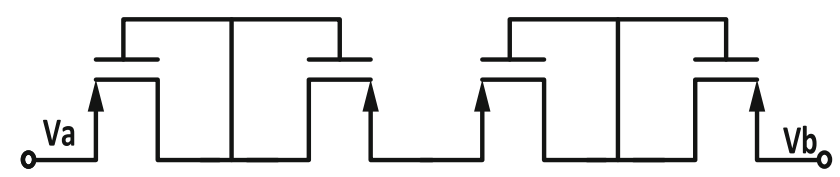

Fig. 6 The circuit of the pseudo-resistor 
Fig. 7 a Circuit diagram of the second order high pass filter, b The frequency response of the high-pass filter

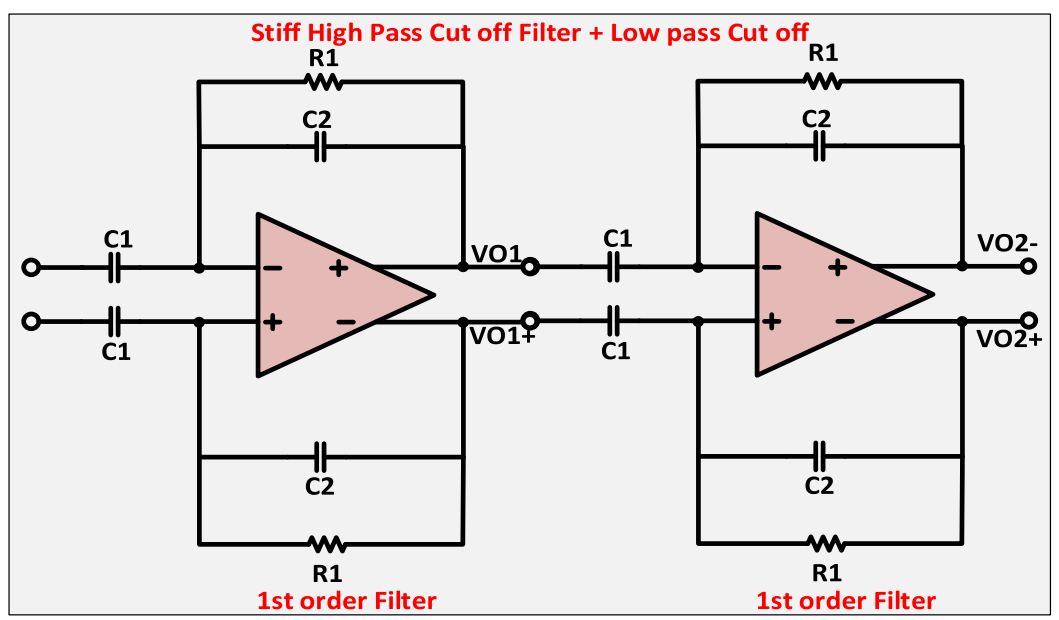

(a)

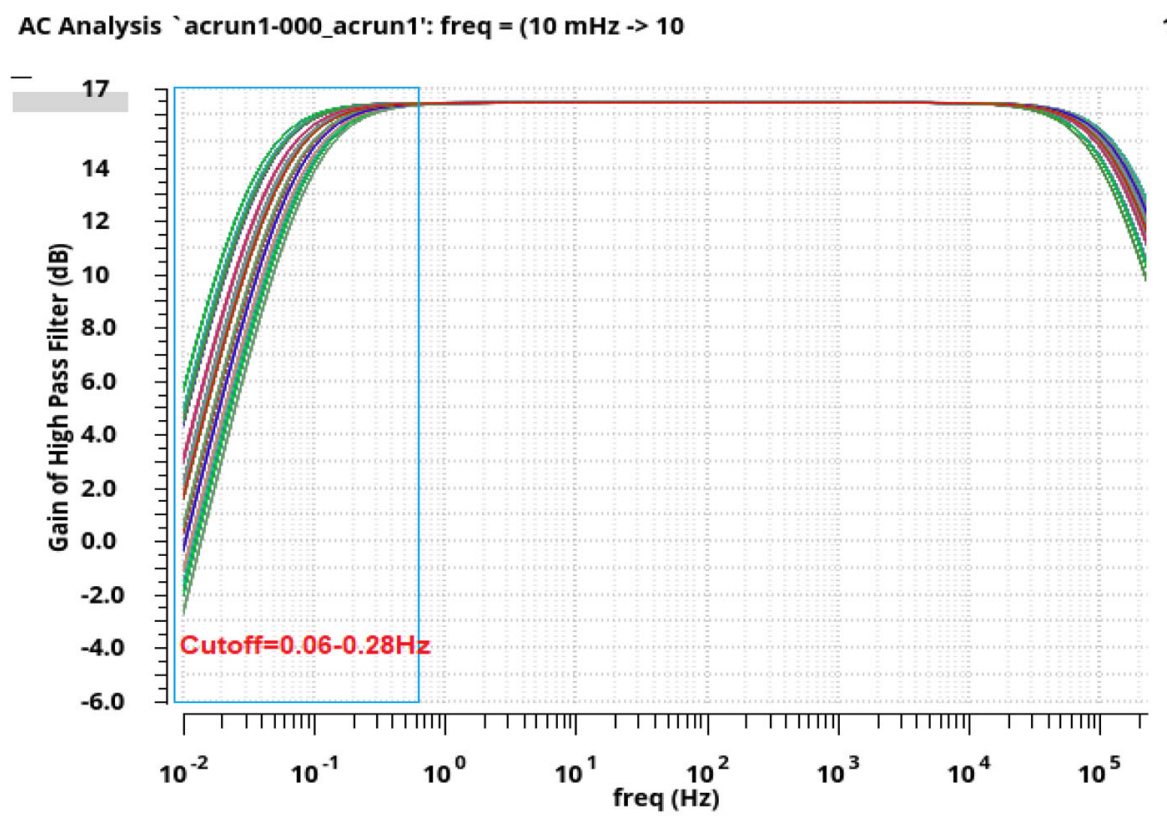

(b) noted that the feedback capacitance $C_{2}$ injects a zero in right-half plane therefore the value of the $C_{2}$ must satisfy the Eq. (3),

$C_{2} \ll \sqrt{C_{1} C_{L}}$

Furthermore, the second order high pass filter is designed by cascading the two first order. The overall circuit diagram of the second order high pass filter and its simulated frequency response is shown in the Fig. 7a and b, respectively.

\subsection{Fourth order low pass $\left(G_{m}-C\right)$ filter}

According to Kao et al. (2018), a high order low pass filter is required to achieved high SNR. As a result, in this study a fourth order, low power, low ripple Butterworth GM-C low-pass filter is employed to achieve the cutoff of $7-$ $25 \mathrm{~Hz}$ (Lee et al. 2009). To achieve the low pass cutoff of $10 \mathrm{~Hz}$, it is well known that the capacitance's accounts for most of the chip area. Therefore, an ultra-low transconductance based low pass filter is designed herein to achieve an ultra-low cut off. The circuit diagram of the low pass filter is shown in Fig. 8, which incorporates two cascaded second order low pass GM-C filter. Each filter incorporates a near-threshold operational transconductance amplifier 


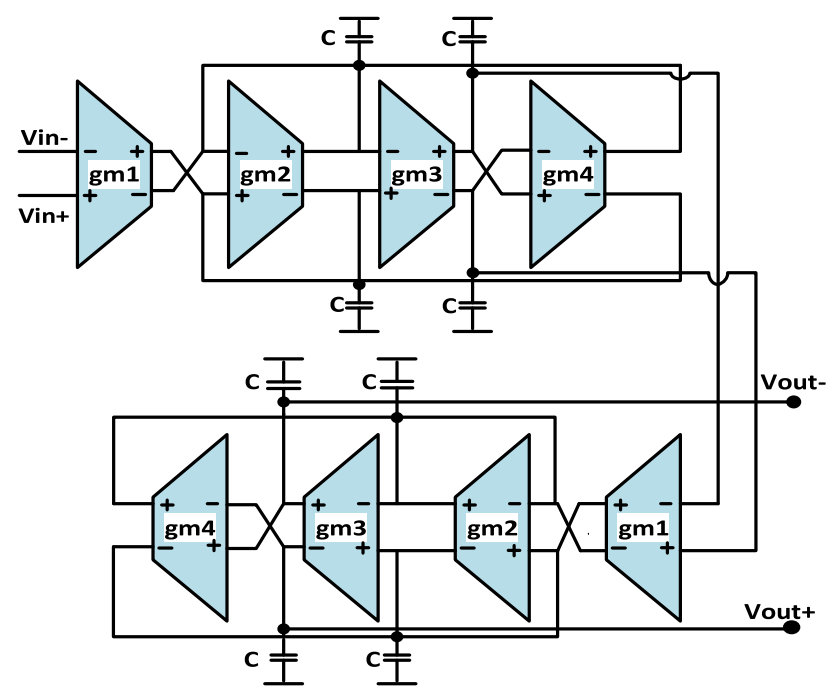

Fig. 8 Fourth order Gm-C low-pass filter circuit

(OTA) and a common mode feedback circuit. The circuit diagram of the OTA circuit and its common-mode feedback circuit are shown in Figs. 9 and 10, respectively. The designed OTA operates in near threshold region. In order to achieve the ultra-low transconductance, the circuit of OTA is shown in Fig. 9, which is design in such a way that, most of current flows to ground through the transistors MM. Thus, the transistor MM, M1 and MN are sized in such a way that the transconductance $\mathrm{gm}_{\mathrm{MM}}>>\mathrm{gm}_{\mathrm{M} 1}, \mathrm{gm}_{\mathrm{MN}}$. On the other hand, the transistor MR operate in triode region and their source-gate voltage is controlled by the transistor MC which makes the trans-conductance less sensitive to common-mode input voltage. Point to be noted that the bias $V_{T}$ is used herein to tune the OTA. In addition, common mode feedback circuit is shown in Fig. 10, which is used to regulate bias voltage $V_{f b}$. The trans-conductance of the designed OTA is given by

$\mathrm{GM}=\frac{i_{o}}{V^{+}-V^{-}}=\frac{N-1}{M+N+1} \cdot g m_{M R}$,

where $\mathrm{M}$ is the ratio of trans-conductance of transistor $M R$ and $M 1$, and $\mathrm{N}$ is the ratio of the transconductance of transistor $M N$ and $M 1$. The transfer function of this second order low-pass filter is

$\mathrm{H}(\mathrm{s})=\frac{V_{\text {out }}}{V_{\text {in }}}=\frac{\frac{g m_{1}}{g m_{4}} \cdot \frac{g m_{3} \cdot g m_{4}}{C_{1} \cdot C_{2}}}{s^{2}+\frac{g m_{2}}{C_{1}} s+\frac{g m_{3} \cdot g m_{4}}{C_{1} \cdot C_{2}}}$

and the simulated frequency response of the low pass filter is shown in Fig. 11. The corner frequency $\left(\omega_{0}^{2}\right)$ and quality factor $(Q)$ is given by

$\omega_{0}^{2}=\frac{g m_{3} \cdot g m_{4}}{C_{1} \cdot C_{2}}, \quad \frac{\omega_{0}}{Q}=\frac{g m_{2}}{C_{1}}$.

Let $g m=g m_{1}=g m_{3}=g m_{4}$ and $C=C_{1}=C_{2}$ lead to,

$\omega_{0}=\frac{g m}{C}, \quad Q=\frac{g m}{g m_{2}}$.

The value of the capacitances $C$ is $10 \mathrm{pF}$. To achieve $-80 \mathrm{~dB} / \mathrm{dec}$, two second order low-pass filters are cascaded in series to form a fourth order filter. The quality factor $(Q)$ of the first and second stage of the filter is 0.54 and 1.31 , respectively. The value of the trans-conductance
Fig. 9 Fully differential OTA circuit for GM-C filter (Lee and Cheng 2009)

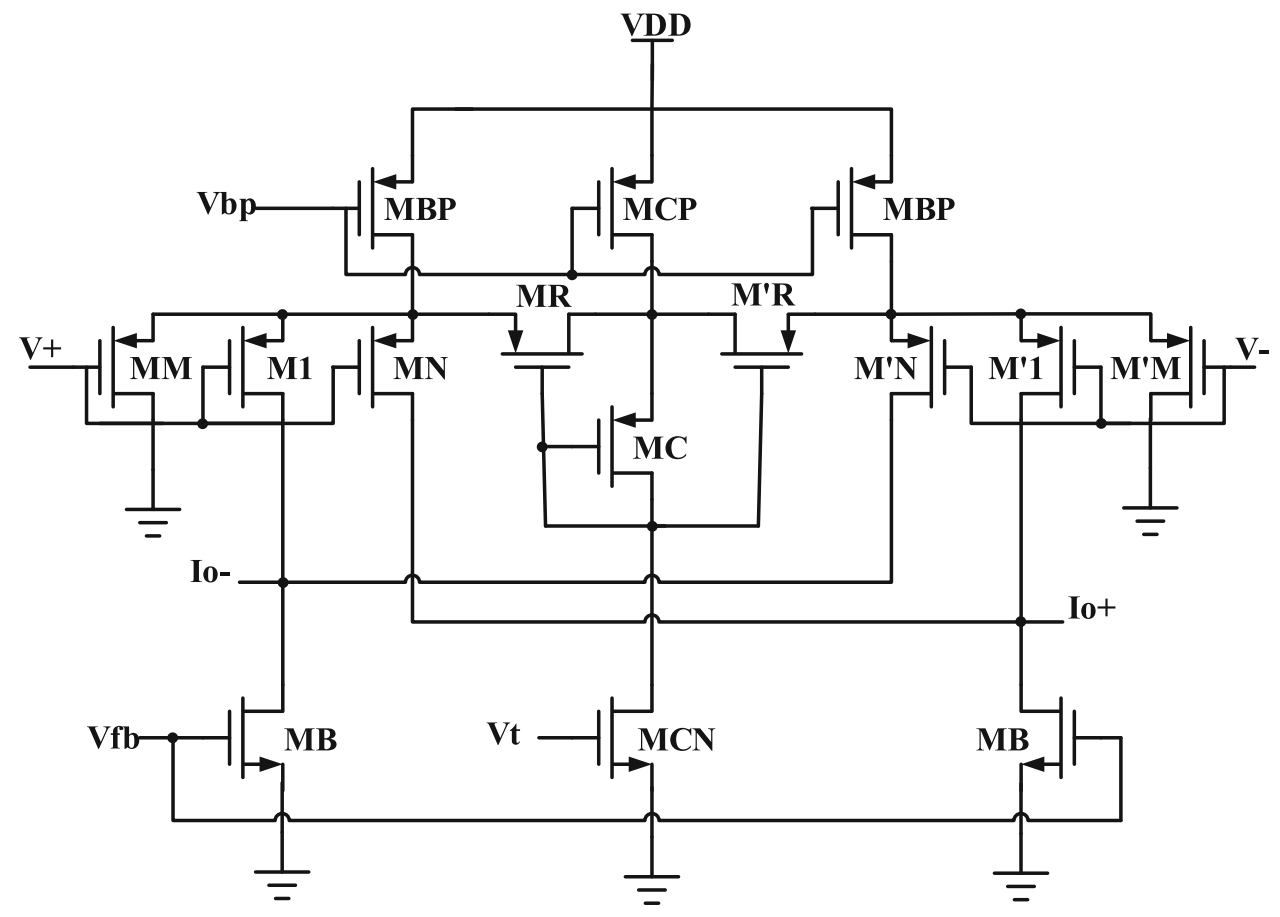




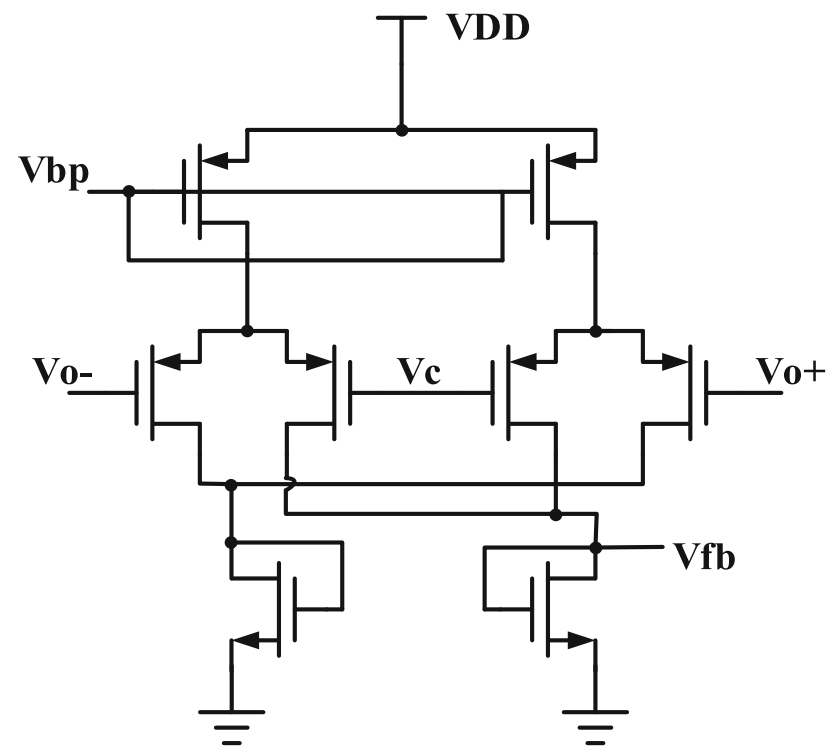

Fig. 10 Common mode feedback circuit

for the stage 1 and stage 2 are $1192 \mathrm{pA} / \mathrm{V}$ and $429 \mathrm{pA} / \mathrm{V}$, respectively.

\subsection{Programmable gain amplifier}

The magnitude of the AC component of the output signal from the filter is too small and also it is different for everyone. Therefore, an appropriate gain adjustment is required to achieve the full dynamic range for the in-built
ADC of the MCU. As a result, a programmable gain amplifier (PGA) circuit is designed, which helps to achieve the full dynamic. The circuit diagram of the PGA is shown in Fig. 12 and it incorporates a cascode amplifier, feedback capacitance transmission gate switches and a 3:8 decoder switch. The 2-bit control signal from the MCU is used herein to control the gain of the PGA by setting the ratio between $C_{i}$ and $C_{f}$. The DC gain of PGA is given by the $C_{\mathrm{i}} /$ $C_{\mathrm{f}}$ and the closed loop gain of the PGA is

$A v=-\frac{C_{i}}{C_{f}} \cdot \frac{1}{1+s \frac{C_{i}}{C_{f}} \cdot \frac{C_{L}}{G_{m}}}$.

The simulated frequency response of the PGA with different gain is shown in Fig. 13, which shows that the achieved gain is $0 \mathrm{~dB}, 2 \mathrm{~dB}, 4 \mathrm{~dB}, 6 \mathrm{~dB}, 8 \mathrm{~dB}, 10 \mathrm{~dB}$, $12 \mathrm{~dB}$ and $20 \mathrm{~dB}$.

\subsection{Power management circuit}

The circuit diagram of the conventional low dropout regulator (LDO) is shown in Fig. 14. The LDO is used herein to supply a fixed voltage to the readout circuit for the stable operation Bandgap reference (BGR) circuit has been design to provide a stable reference voltage to the PPG readout circuit and LDO. BGR circuit ensures the sufficient power supply noise rejection from the supply voltage and temperature variation. The circuit diagram of the subthreshold bandgap reference circuit (Pandey et. al 2020) is shown in Fig. 15. The principle of bandgap reference is
Fig. 11 The frequency response of the low-pass filter

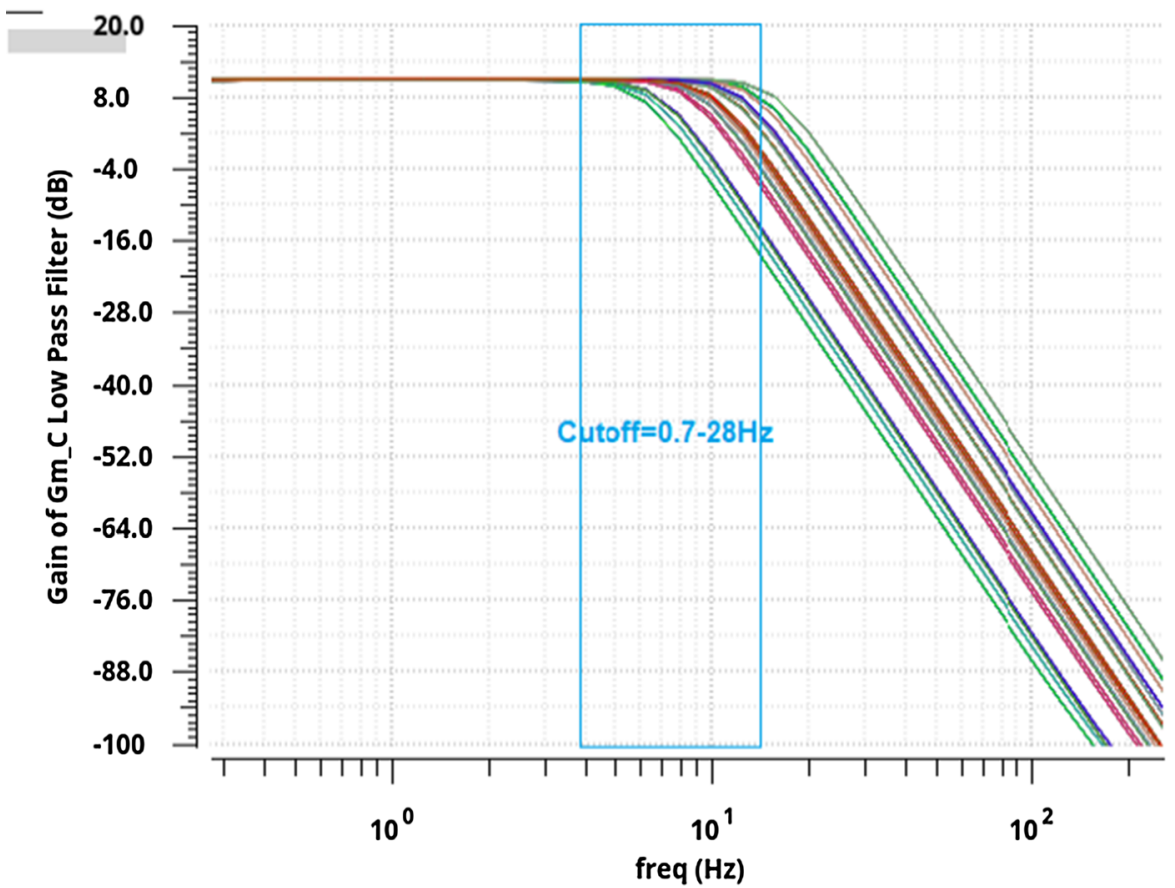


Fig. 12 Programmable gain amplifier circuit

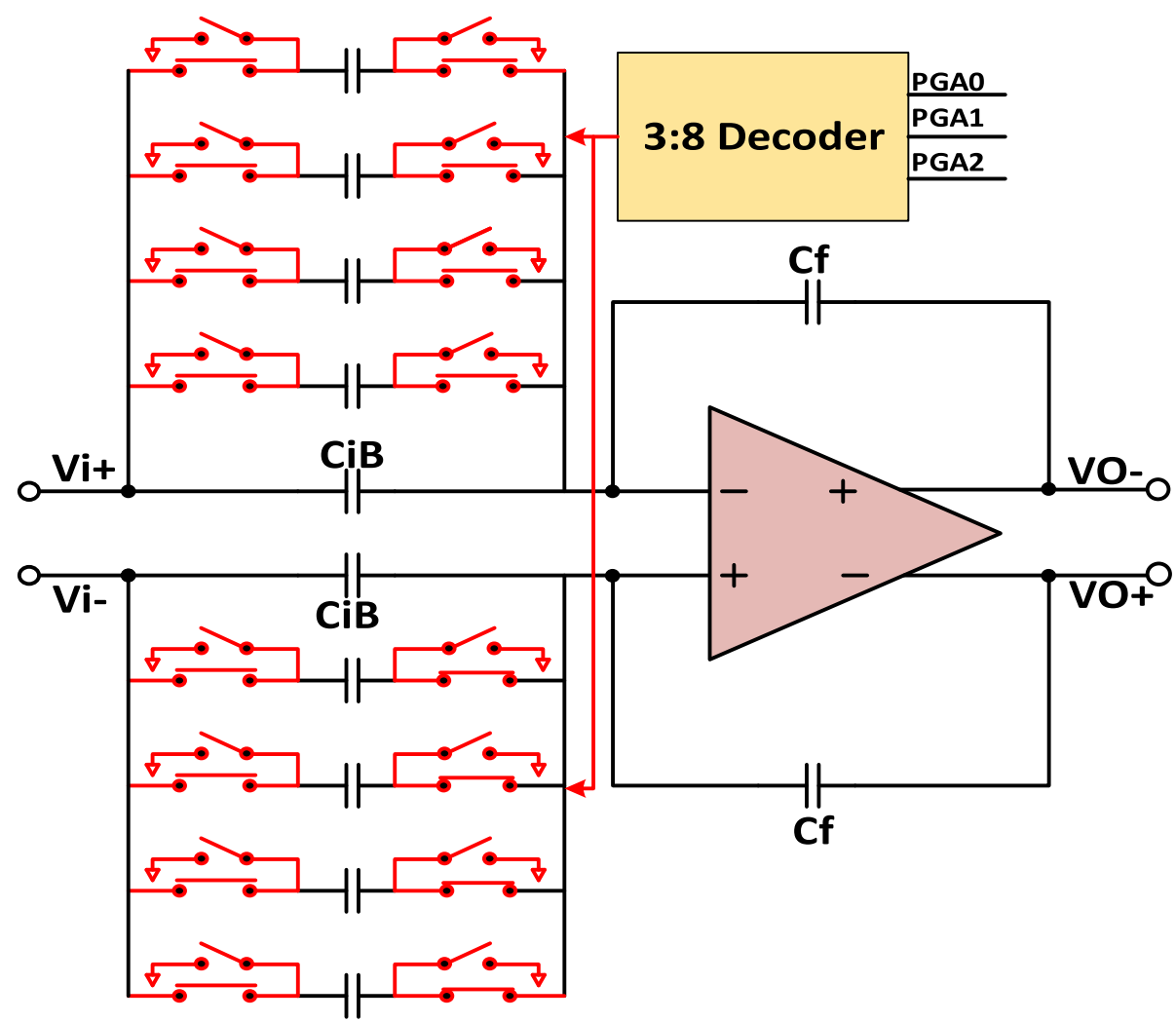

AC Analysis 'acrun1-000_acrun1': freq $=(100 \mathrm{mHz} \rightarrow 100$

1

Fig. 13 Frequency response of the PGA@ different gain based on the proportional to absolute temperature current (PTAT) and a complementary to absolute temperature current (CTAT). In this circuit, the quiescent current is less than $5 \mu \mathrm{A}$. The output voltage can be derived, yielding
$V_{r e f}=\left[\frac{P_{10}}{P_{7}} I_{A}+\frac{P_{11}}{P_{2}}\left(\frac{V_{G S 3}}{R_{1}}-N I_{A}\right)\right] \times R_{3}$,

where $P=\frac{W_{e f f}}{L_{e f f}}, \mathrm{~N}$ is the multiple of current $\mathrm{I}_{\mathrm{A}}$. 


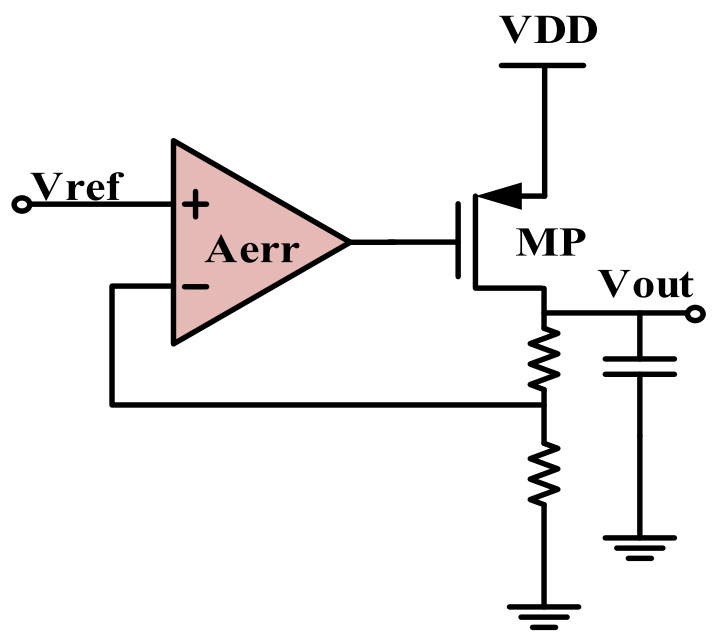

Fig. 14 The conventional low dropout regulator (LDO)

\subsection{OLED driver circuitry}

A PWM-DAC based OLED driver is shown in Fig. 16, which is used to control the intensity of the OLED. Analog multiplexer/demultiplexer has been used to select one of the OLED from $2 \times 2$ array. In this study a ring oscillatorbased, voltage-controlled oscillator (VCO) with one-bit current mode digital to analog converter has been used to control the intensity of the OLED. Based on the output from the readout circuit, MCU is used to control the VCO output. The output of the VCO is the pulse width modulated signal (PWM). Therefore the, the duty cycle of the PWM directly modulates bias current of the DAC. Point to be noted that the duty cycle of the PWM is directly proportional to the intensity of the OLED. For the real time feedback, MCU is acts as a bridge between readout system

Fig. 15 The band gap reference circuit
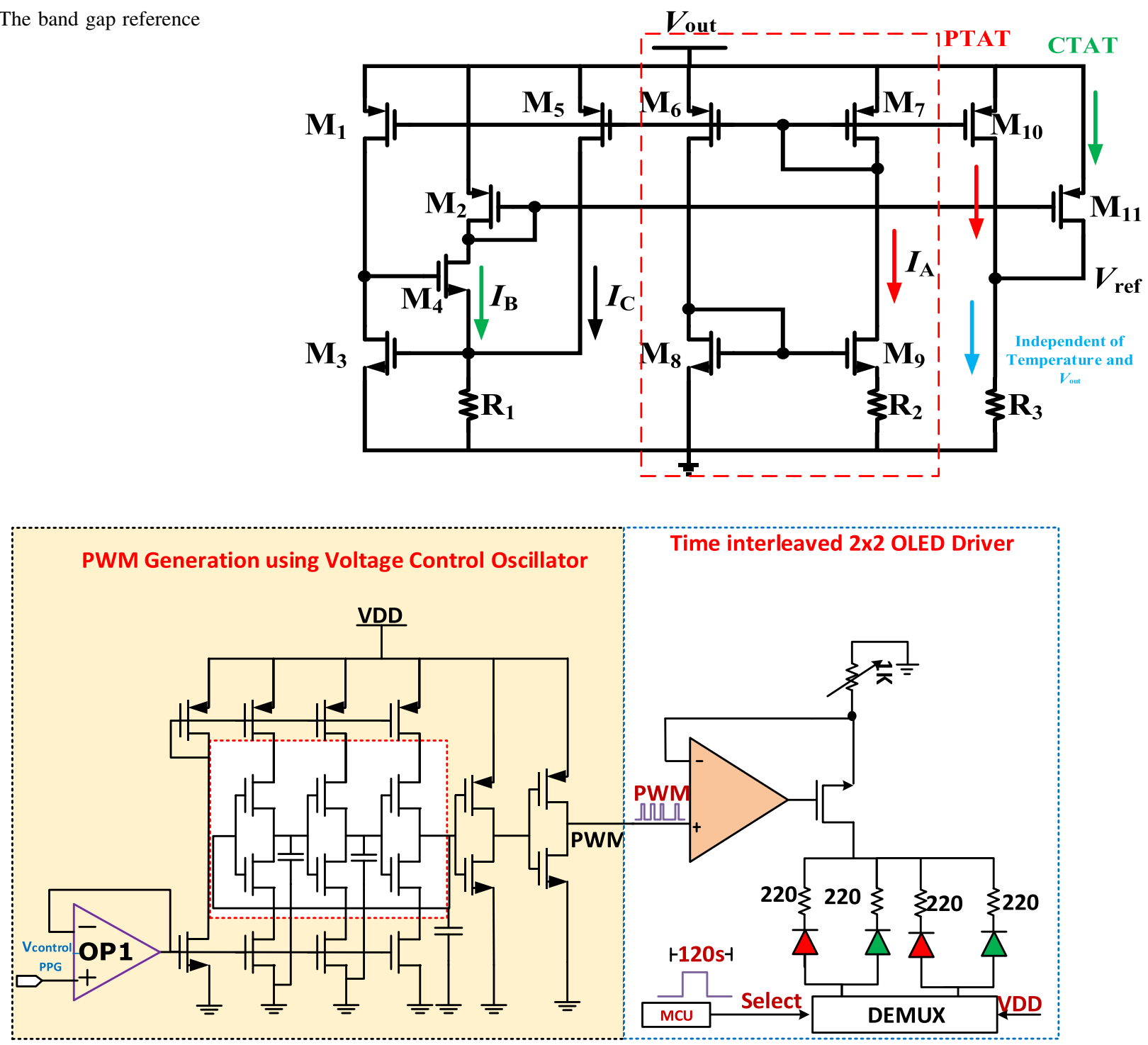

Fig. 16 PWM-DAC based Intensity control and multi-OLED selection Driver 
Fig. 17 Principle of the time interleaved OLED array control
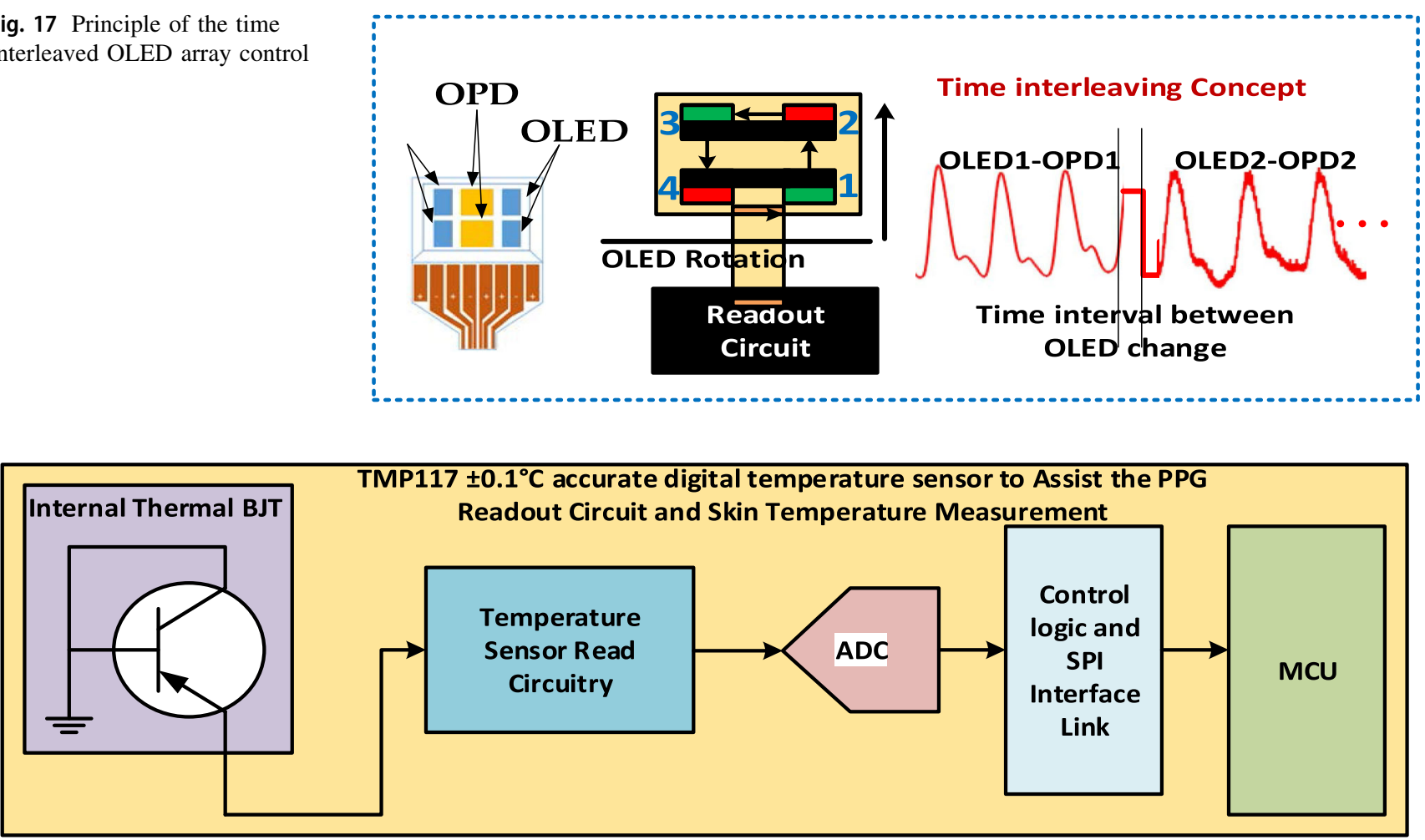

(a)

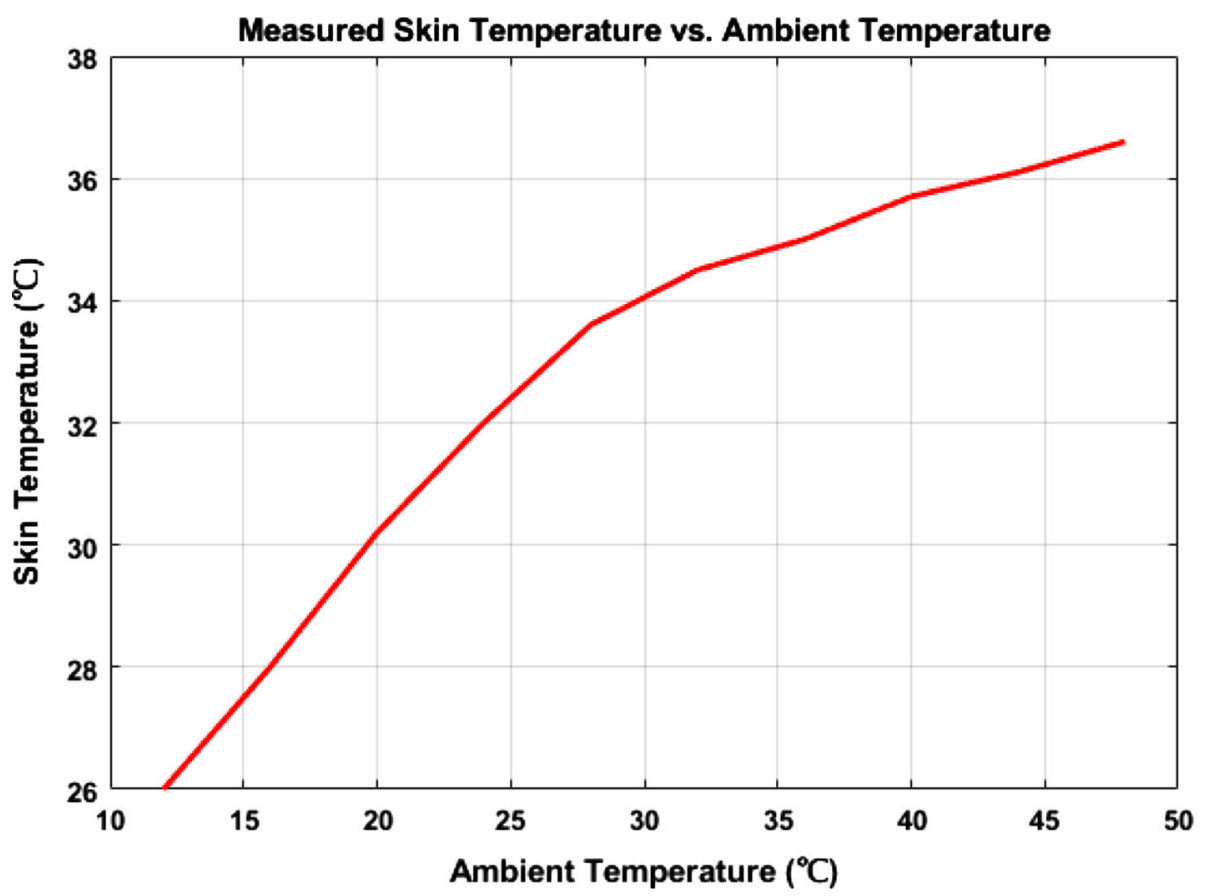

(b)

Fig. 18 a Block diagram of the temperature sensor used with the PPG sensor readout, $\mathbf{b}$ The measured average skin temperature with respect to the ambient temperature

and external software (MATLAB), via the Bluetooth transceiver. The concept of the time interleaved is shown in Fig. 17. Time-interleaved concept used herein to minimize the mispositioning in such a way that a the 2-OPDs are connect to the single readout channel then each of the $2 \times 2$ OLEDs are rotated for $2 \mathrm{~s}$. After this, PPG signal 
from each OLED are scanned and tested for the quality index (will be discussed in Subsect. 2.10). The OLED, which shows the highest quality factor, that will be assign for the bio-sign measurement until the next scan phase will arrive. MATLAB based control system is used herein via the Bluetooth transceiver. For the portability, android APP and APP server has been developed to provide remote interface via TCP/IP.

\subsection{Temperature sensor}

The block diagram of the high-precision digital temperature sensor (TMP117) is shown in Fig. 18a. It is designed to meet the ASTM E1112 and ISO80601 requirements for electronic patient thermometers. This resolution of the 16-bit temperature sensor is $0.0078{ }^{\circ} \mathrm{C}$. Without using any calibration, this sensor can achieve an accuracy of \pm $0.1{ }^{\circ} \mathrm{C}$ across the temperature range of -20 to $50{ }^{\circ} \mathrm{C}$. The $\mathrm{I}^{2} \mathrm{C}$ interface is used for the peripheral communication. The peak power consumption of the TMP117 IC is $3.5 \mu \mathrm{A}$. The integrated temperature sensor is used to measure the skin temperature parallel with the PPG signal. The measured skin temperature with respect to the ambient temperature is shown in Fig. 18b. This data has been used for the temperature compensation to improve the PPG quality at the low ambient temperature. In addition to this also helps to enhance the measurement accuracy of the $\mathrm{HR}, \mathrm{S}_{\mathrm{p}} \mathrm{O}_{2}$ and BP.

\subsection{Digital control mechanism and temperature compensation}

The algorithm to adjust the gain and intensity is shown in Fig. 19. MATLAB based control system is used to interface with readout $+\mathrm{MCU}$ via the Bluetooth transceiver. After receiving the PPG signal, the control system firstly checks the skin temperature. Point to be noted that the green and red OLED has been used here and the wavelength is just limited to $530 \mathrm{~nm}$ and $630 \mathrm{~nm}$. If the skintemperature reduces down below the $32{ }^{\circ} \mathrm{C}$. In this case, the light penetration depth $(\boldsymbol{\delta})$ in skin is increased. Therefore, the emitted light goes deeper and the reflected wave PPG is unable to reached the OPD through banana path. Thus, decreasing the intensity minimize the incidence plus the reflection path. Thus, the light will not go deeper can easily reflect back from the microvascular layer behind the skin. Therefore, MCU will first follow the temperature compensation algorithm as shown in Fig. 20 to obtained a quality PPG signal from the wrist artery. On the other hand, in case, if the temperature of the skin is in the normal range $\left(25-38{ }^{\circ} \mathrm{C}\right), \mathrm{MCU}$ will adjust the intensity and gain of the readout circuit as per the quality index. The quality evaluation and compensation algorithm are shown in Fig. 19.
Signal saturation, outlier, power spectrum (FFT), periodicity and $\mathrm{AC} / \mathrm{DC}$ ratio is used as the signal quality index. In order to pass the quality, the receive signal should be

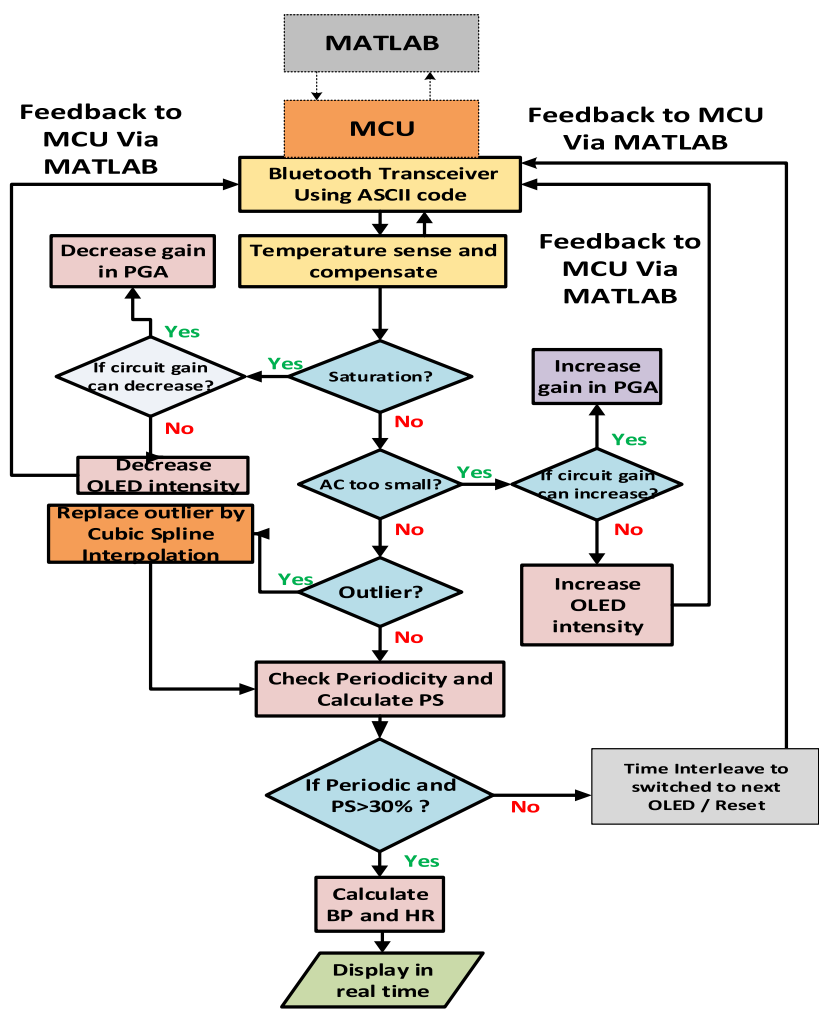

Fig. 19 Signal quality check and digital control strategy

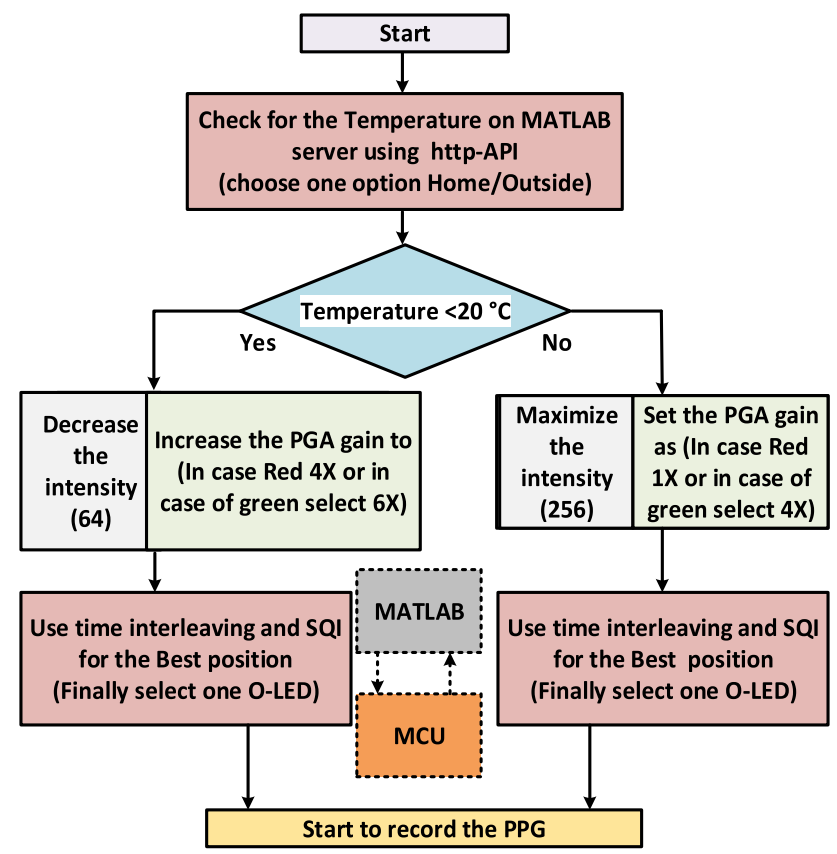

Fig. 20 The digital adjustment-control for temperature compensation and misposition minimization (Pandey et al. 2019) 


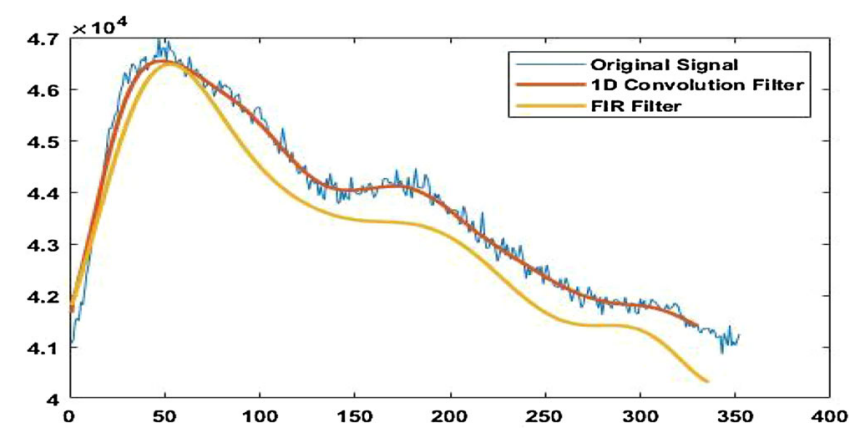

(a)

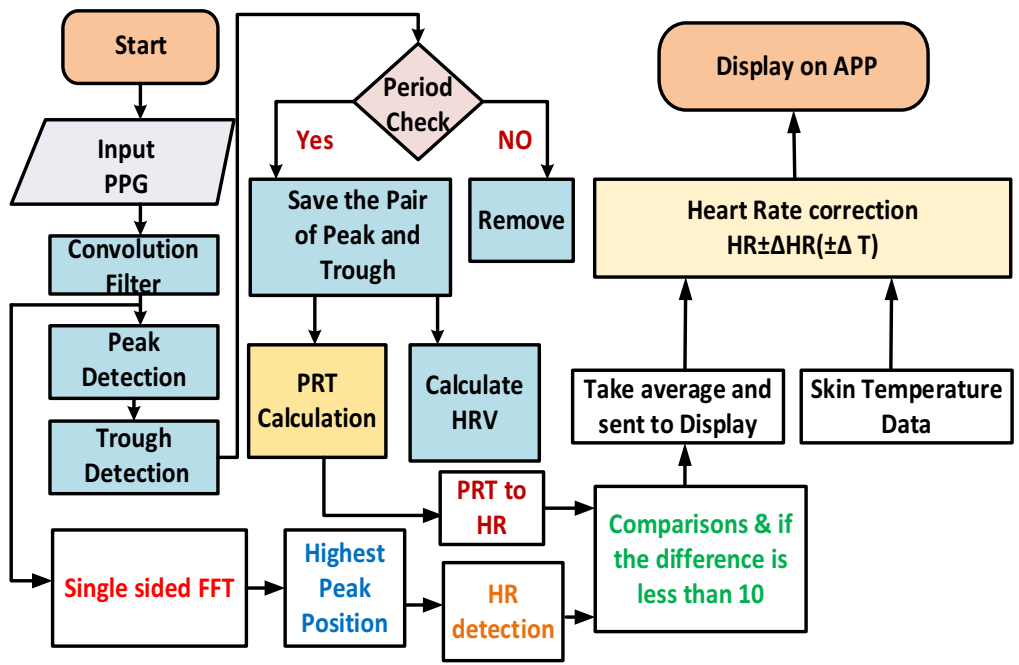

(b)
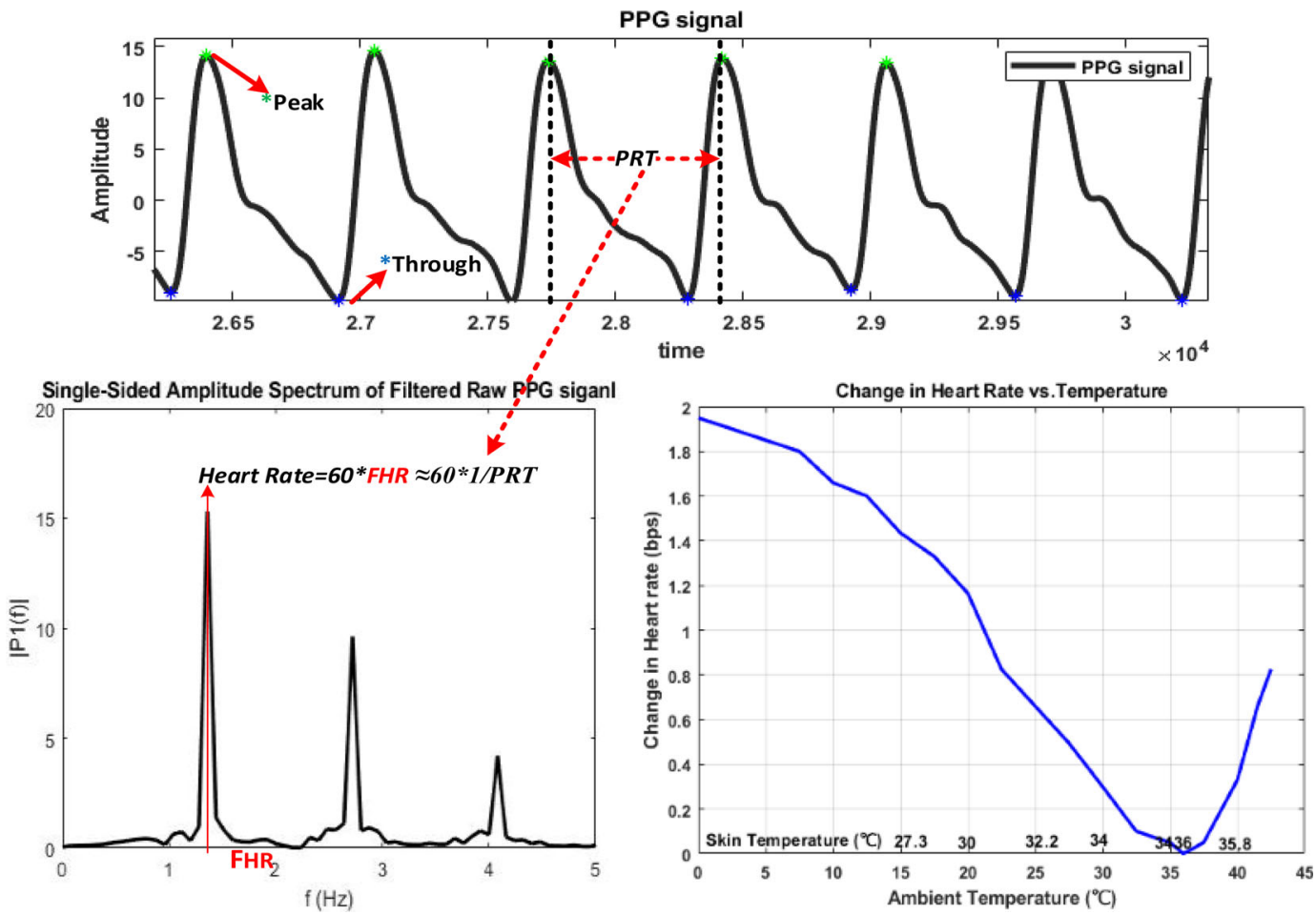

(c) 
4Fig. 21 a Output of the convolution based moving average filter and comparison, b HR measurement algorithm. c The obtained output PPG signal, its power spectrum, and HR variation with temperature change

periodic, the peak amplitude must be greater than $0.9 \mathrm{~V}$, and the power spectrum percentage (PS) should be greater than the $30 \%$. Here, periodicity is measured in terms of the ratio of the period of two cycle of the of the PPG signal. If the ratio of peak to peak time (PRT, Fig. 21c) of two cycle of the PPG signal is in the range of 0.9-1.1 that means the signal is periodic (Fig. $21 \mathrm{c}, 0.9<\frac{P R T 1}{P R T 2}<1.1$ ). PS is calculated by the ratio of the sum of the magnitude of the first two harmonics to the sum of all the harmonics in the FFT of the PPG signal. In order to use this algorithm and to resolve the issue related to temperature, skin color and tissue texture, it is suggested to start the calibration scanning process from the high intensity to low intensity and low gain to high gain simultaneously (e.g. OLED 100\% duty cycle and PGA $=0 \mathrm{~dB}$, then OLED 90\% duty cycle and $\mathrm{PGA}=2 \mathrm{~dB}$ ).

\section{Pre-signal processing and bio-signs detection}

\subsection{Pre-signal processing and heart rate estimation}

The weighted convolution-based time domain filtering is used herein to enhance the signal to noise ratio (SNR) of the PPG signal. The principle of weighted filter is based on the least square quadratic smoothing. If " $x$ " is data set and " $f$ " is the filter weight. Then after convolving $x$ and $f$, the $x$ ' would be the smoothed data at the time instant $t$. The dot product of the data and filter weight ( $\mathrm{n}$ is the size of the filter) is given by

$f \cdot x=\sum_{i=1}^{n} f_{i} \cdot x_{i}$.

Similarly, the upcoming smoothing data set would be

$x_{m}^{\prime}=\frac{\left(\sum_{i=1}^{n} f_{i} \cdot x_{i+1}\right)}{\sum_{i=1}^{n} f_{i}}$.

Therefore, the response of the convolution based moving average filter and its comparison with FIR filter is shown in Fig. 21a. The filter response shows that the convolution-based time domain filter is much better than the FIR based low pass digital filter. The algorithm to estimate the HR is shown in Fig. 22b. After filtering, peak and the valley detection is performed. If the receive PPG signal is a periodic signal, the pairs of peak and valley will be identified, then HR will be calculated. As discussed earlier Periodicity, and PS (single sided FFT) of the PPG is used herein to estimate the signal quality index. The feature from the time domain and frequency domain are shown Fig. 22c.

After detecting the peak and through, the peak-peak interval/pulse repetition time (PRT) is measured. At the same time, the first harmonics of the single sided spectrum of the received PPG signal is used to calculated the the frequency (FHR). Therefore, the heart rate in time domain and frequency domain can be related by the equation given below

$$
\begin{aligned}
\operatorname{HeartRate}(H R) & =60 * F H R \\
& =60 *\left(\frac{1}{P R T}\right) \pm \Delta H R( \pm \Delta T) .
\end{aligned}
$$

Usually the normal temperature of the body is $37^{\circ} \mathrm{C}$. However, as per this study skin temperature of the $35{ }^{\circ} \mathrm{C}$ is taken as the threshold temperature. In this study, it is observe that for every $1{ }^{\circ} \mathrm{C}$ decrease in the mean ambient temperature below the threshold temperature tends to 0.06 beats/min in HR. Similarly, for every $1{ }^{\circ} \mathrm{C}$ increase in the mean ambient temperature above the threshold temperature tends to 0.13 beats $/ \mathrm{min}$, in HR. Therefore, the term $\pm \Delta H R( \pm \Delta T)$ is used as the correction value to the calculated HR.

\subsection{Blood oxygen saturation estimation}

Oxy-hemoglobin $\left(\mathrm{Hbo}_{2}\right)$ and deoxy-hemoglobin $(\mathrm{Hb})$ have different absorptivity's at red and green wavelengths, as highlighted on the absorptivity of oxygenated and deoxygenated hemoglobin as mention in (Lochner et al. 2014). The blood oxygen saturation $\left(\mathrm{SO}_{2}\right)$ is quantified according to Eq. (13). Here, $\mathrm{C}_{\mathrm{Hbo}_{2}}$ and $\mathrm{C}_{\mathrm{Hb}}$ are the concentrations of oxy-hemoglobin and deoxy-hemoglobin, respectively.

$\mathrm{SO}_{2}=\frac{\mathrm{C}_{\mathrm{Hbo}_{2}}}{\mathrm{C}_{\mathrm{Hbo}}+\mathrm{C}_{\mathrm{Hb}}}$,

$R_{O S}=\frac{A_{\text {red }}}{A_{\text {green }}}=\frac{\ln \left(T_{n, \text { red }}\right)}{\ln \left(T_{n, \text { green }}\right)}$,

$R_{O S}$, the ratio of absorbed red $\left(A_{\text {red }}\right)$ and green $\left(A_{\text {green }}\right)$ light, depends on the normalized transmitted red $\left(T_{n, \text { red }}\right)$ and green $\left(T_{n, \text { green }}\right)$. light intensities. Finally, arterial oxygen saturation $\left(\mathrm{S}_{a} \mathrm{O}_{2}\right)$ can be calculated using Eq. 15 . 


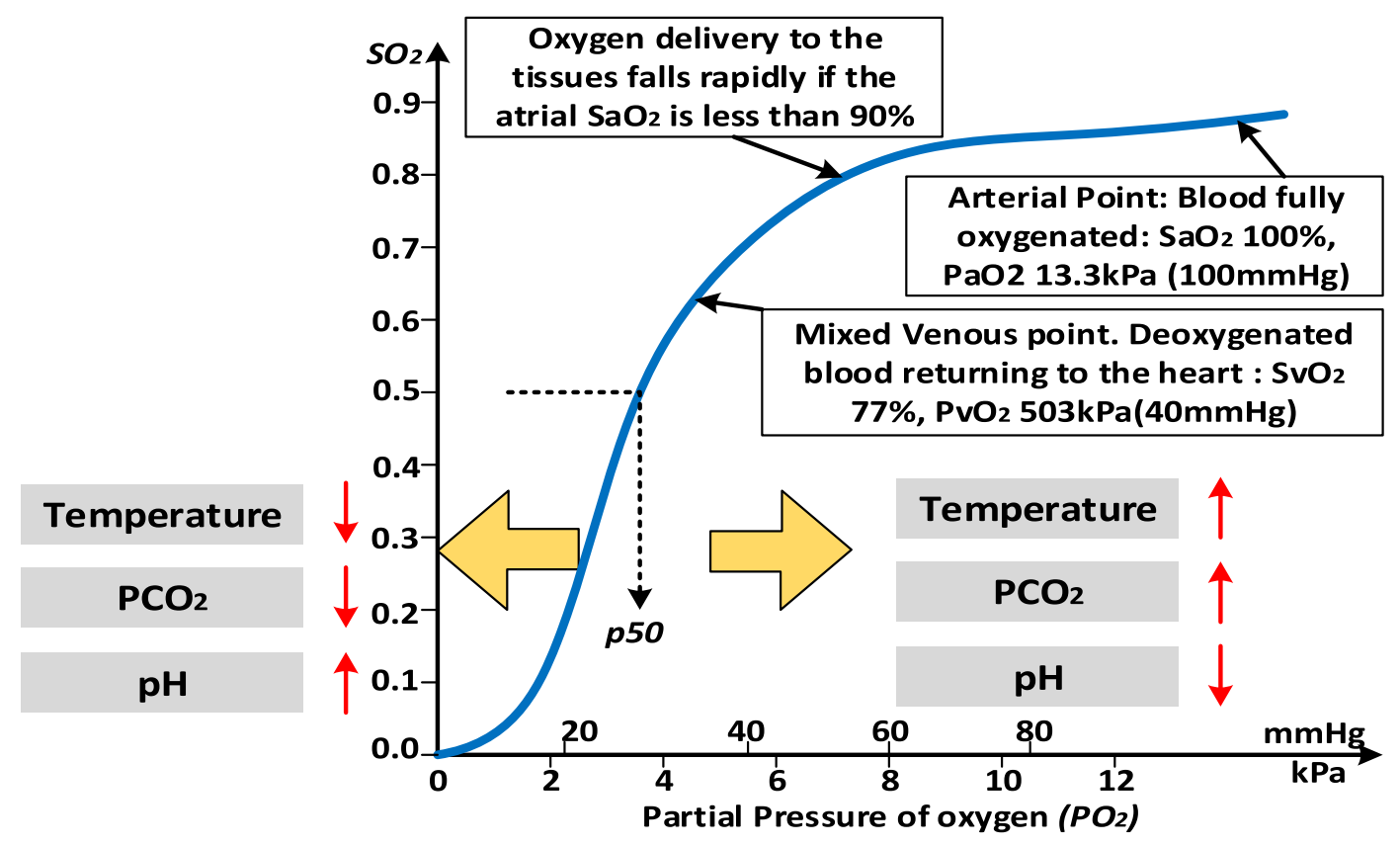

(a)

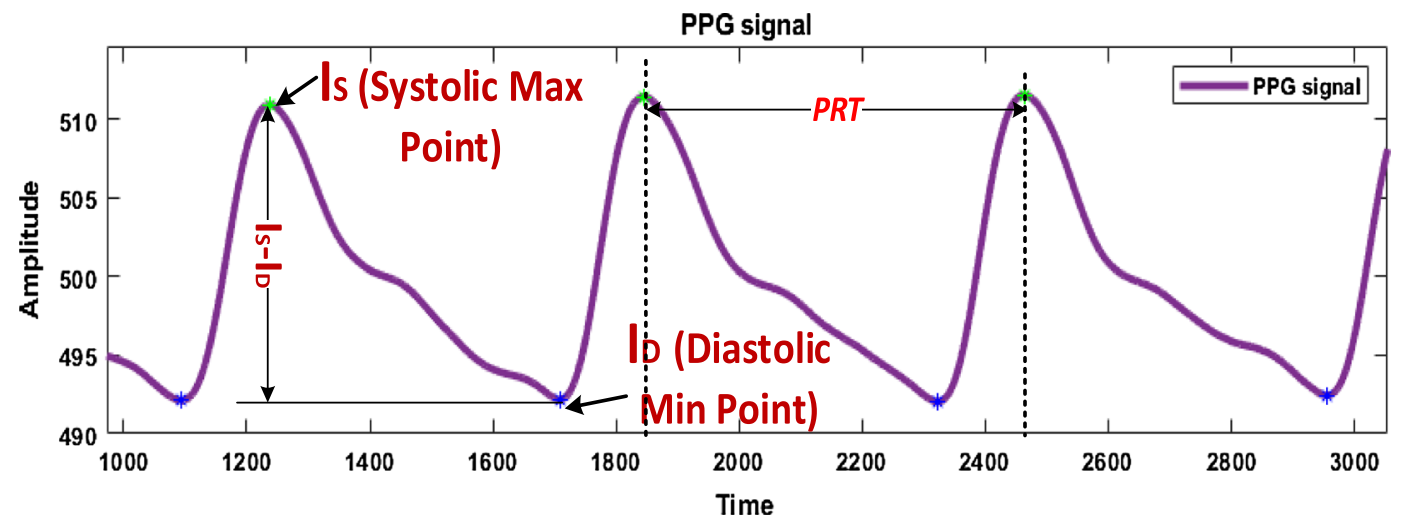

(b)
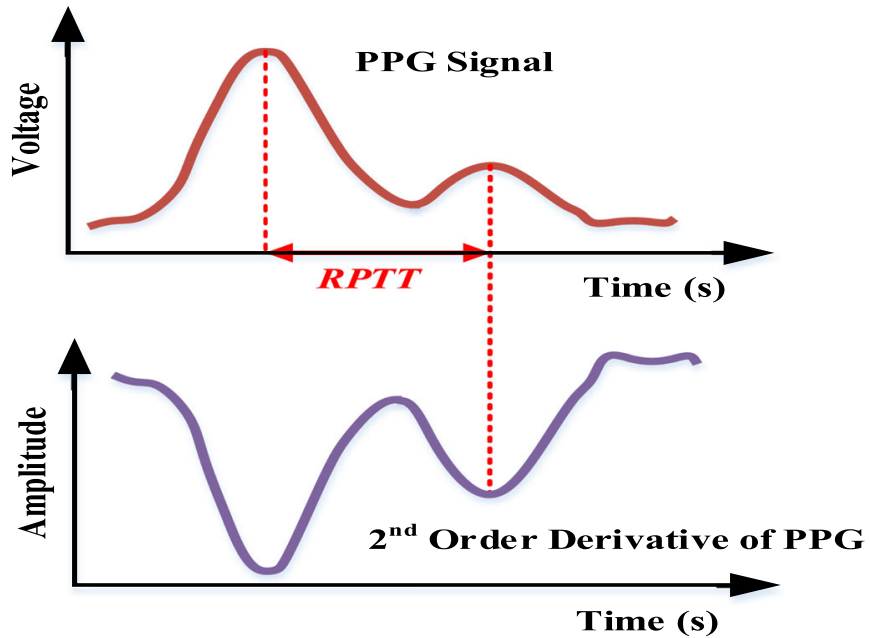

(c) 
4Fig. 22 a Oxygen dissociation curve (ODC), b systolic maximum and diastolic minimum point detection, and c method to estimate repulsive transmit time (RPTT)

$$
\begin{aligned}
S_{a} O_{2}\left(R_{O S}\right)= & \frac{\varepsilon_{\text {redHb }}-\varepsilon_{\text {green } H b} R_{O S}}{\left(\varepsilon_{\text {redHb }}-\varepsilon_{\text {redHbo } 2}\right)+\left(\varepsilon_{\text {green } H b O 2}-\varepsilon_{\text {green } H b}\right) R_{O S}} \\
& \pm \Delta S_{a} O_{2}( \pm \Delta T),
\end{aligned}
$$

where $\varepsilon_{\text {red,Hb }}$ and $\varepsilon_{\text {green,Hb }}$ are the molar absorptivity of deoxy-hemoglobin at red $(\lambda=630 \mathrm{~nm})$ and green $(\lambda=530 \mathrm{~nm})$ wavelengths, respectively. Similarly, $\varepsilon_{\text {redHbo } 2}$ and $\varepsilon_{\text {greenHbO2 }}$ are the molar absorptivity of oxy-hemoglobin at red $(\lambda=630 \mathrm{~nm})$ and green $(\lambda=530 \mathrm{~nm})$ wavelengths, respectively. The value of the $\varepsilon_{\mathrm{Hb}}$ and $\varepsilon_{\mathrm{Hb}}$ are taken from the (Prahal 1999). The standard ODC curve (body temperature $37^{\circ} \mathrm{C}$ ) is shown in Fig. 23a, which shows that the sigmoid relationship between the partial pressure of oxygen and the oxygen saturation. If the curve is shifted to the right that means the body temperature is increase; and if the curve is shifted to left that means the body temperature increase. However, temperature does not have so serious effect but hyperthermia causes a rightward shift, while hypothermia causes a leftward shift. Therefore, the term $\Delta S_{a} O_{2}( \pm \Delta T)$ as mention in the Eq. 15 denotes the change in blood oxygen due to change in the temperature (hyperthermia/ hypothermia). Point to be noted that the $\mathrm{S}_{a} \mathrm{O}_{2}$ is oxygen saturation of arterial blood, while $\mathrm{S}_{P} \mathrm{O}_{2}$ is oxygen saturation as detected by the pulse oximeter. They are called arterial blood oxygen saturation and percutaneous oxygen saturation, respectively. Therefore, in order to calculate the accurate $S_{P} O_{2}$, from the designed PPG acquisition system, the $\mathrm{S}_{P} \mathrm{O}_{2}$ measurement is calibrated as follows:

$R_{O S}=\frac{\left[\left(I_{D}-I_{S}\right) / I_{S}\right)_{\text {red }}}{\left[\left(I_{D}-I_{S}\right) / I_{S}\right)_{\text {green }}}$,

Fig. 23 Blood pressure measurement algorithm

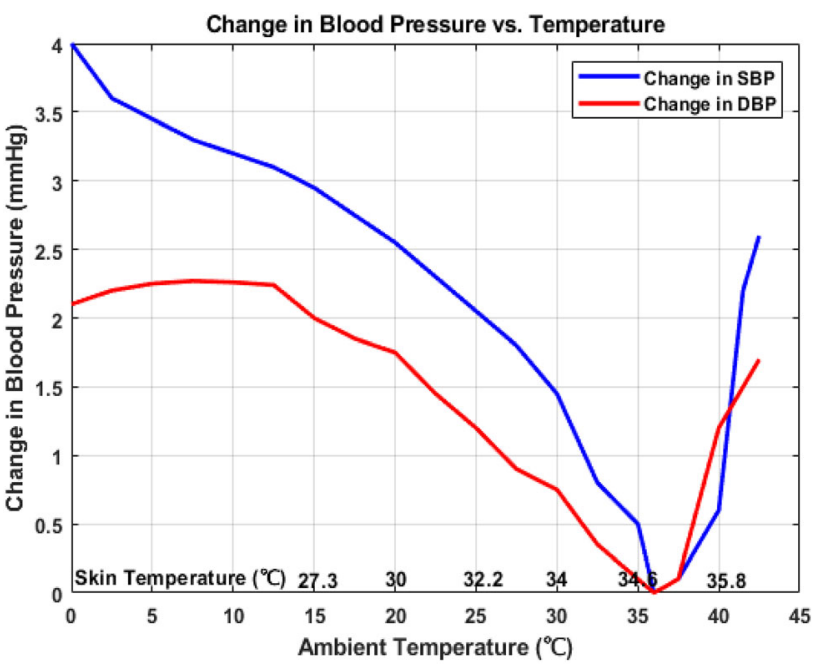

Fig. 24 Plot for the change in SBP and DBP vs temperature

where $I_{D}$ is the value of the PPG pulse at end-diastole and $I_{S}$ is the minimum value of the PPG pulse during systole. As shown in Fig. 23b, the value of $I_{D}$ (for the determination of the pulse amplitude, $I_{D}-I_{S}$ ) was taken as the value of the extrapolated line connecting the two maxima of the pulse at the time of pulse minimum. The pulse amplitude was taken as the difference between the extrapolated value of $I_{D}$ and the measured value of $I_{S}$. The measured $S_{P} O_{2}$ in the range of 95-100\% denotes the acceptable normal ranges, $92 \%$ or less refer for oxygen assessment, 88-94\% denotes hypoxic drive problem, and $\mathrm{S}_{P} \mathrm{O}_{2} 100 \%$ probably indicates carbon-monoxide poisoning or cyanide poisoning.

\subsection{Blood pressure estimation}

According to Bramwell-Hill equation (Bramwell et al. 1992), the pulse wave velocity (PWV) inside the blood vessel is directly depend on the BP (SBP and DBP), the
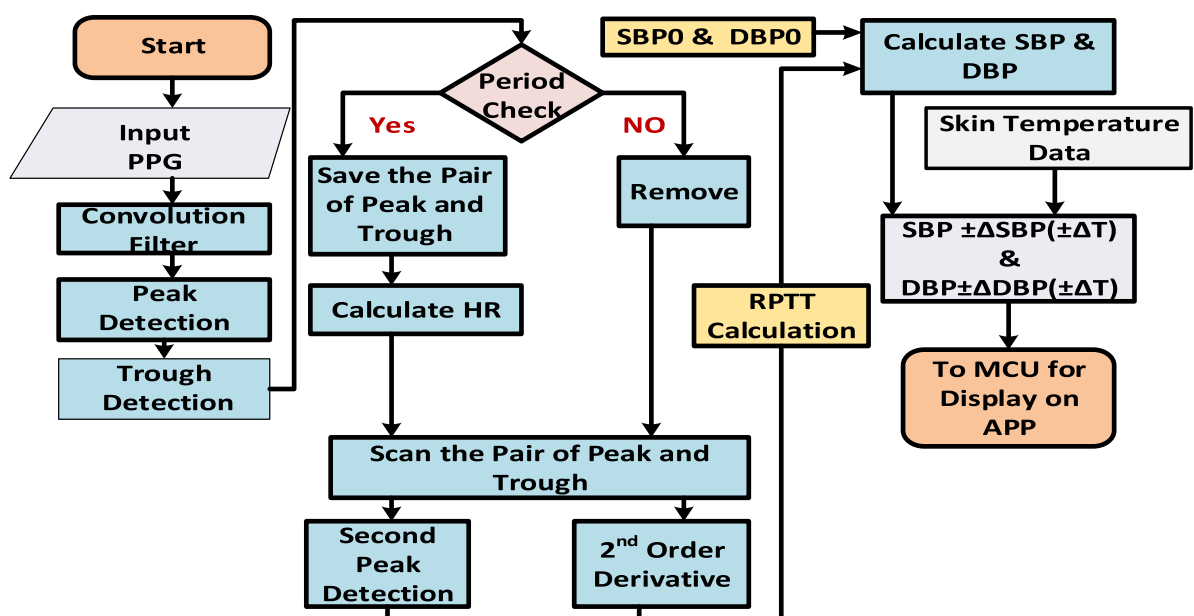
Fig. 25 PSRR of whole readout circuit with five process corners

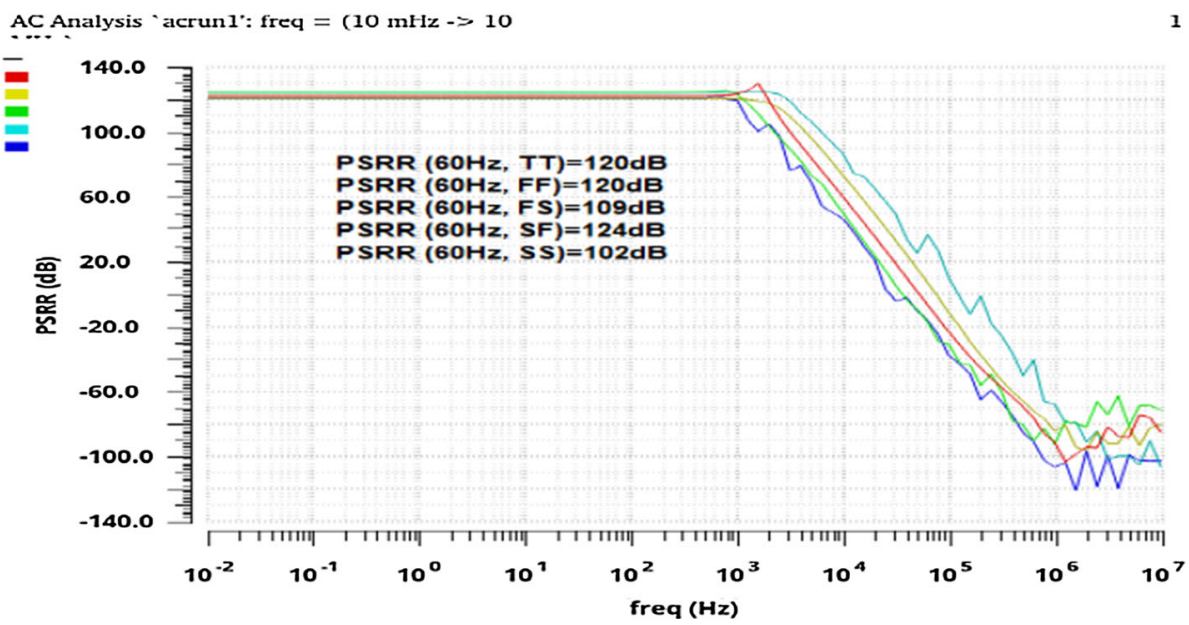

Fig. 26 Noise simulation of whole readout circuit with five process corners

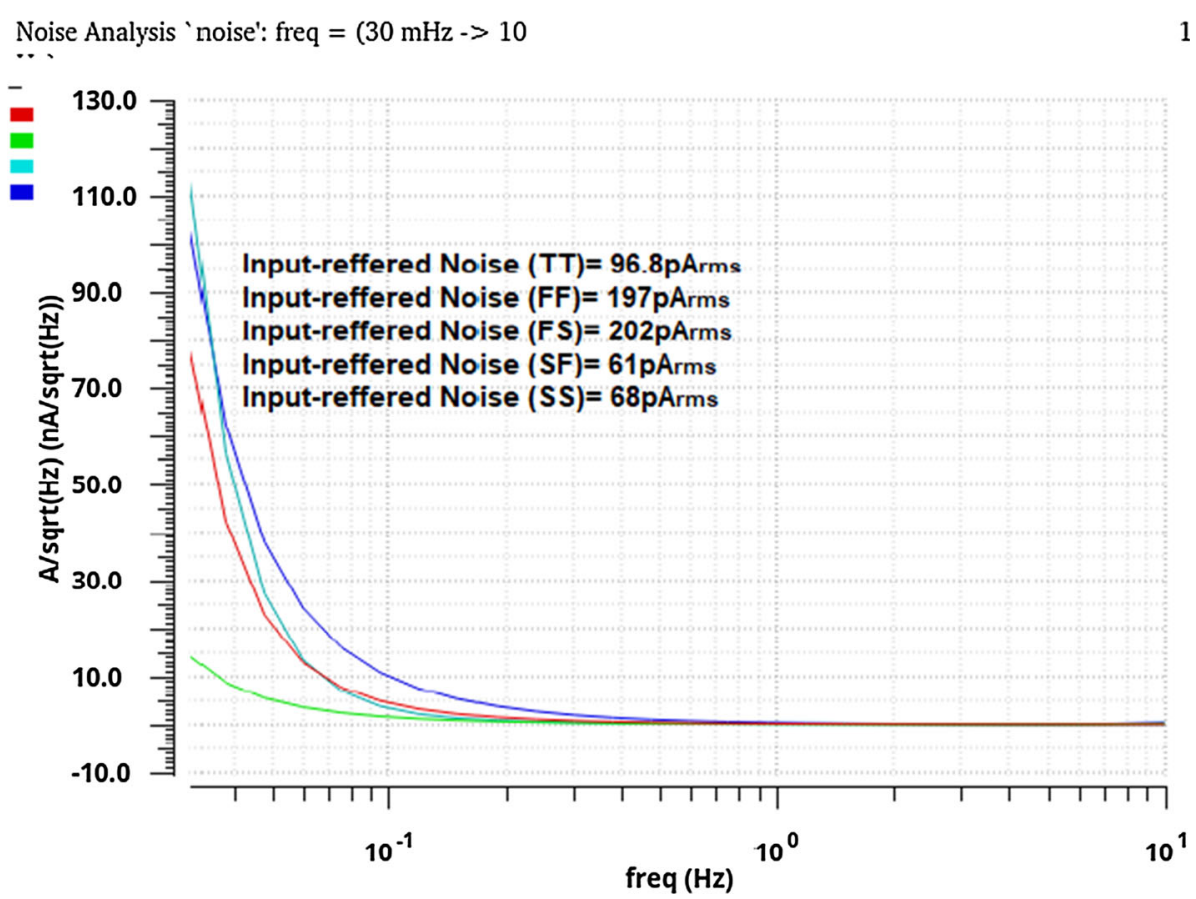

density of blood ( $\rho$ ), and the volume of blood in artery (V). Therefore, the equation for the PWV can be modelled as,

$\mathrm{PWV}=\sqrt{\frac{\mathrm{V}}{\rho}\left(\frac{\mathrm{SBP}-\mathrm{DBP}}{\Delta \mathrm{V}}\right)}=\frac{L}{R P T T}$,

where $\mathrm{L}$ is a local distance along the aorta from measured point to lower body. The blood density, the blood volume in artery, and the change in blood volume are closely related to the PPG incident and reflected wae, therefore the Eq. (17) can be simplified to

$\mathrm{SBP}-\mathrm{DBP}=\frac{\rho \Delta \mathrm{V}}{\mathrm{V}}\left(\frac{\mathrm{L}}{\mathrm{RPTT}}\right)^{2}=\mathrm{K}_{\mathrm{a}}\left(\frac{1}{\mathrm{RPTT}^{2}}\right)$,
$M B P=K_{b}+\left(\frac{2}{0.031}\right) \ln \left(\frac{K_{c}}{R P T T}\right)$,

$M B P=\left(\frac{1}{3}\right) S B P+\left(\frac{2}{3}\right) D B P$,

$D B P=K_{b}+\left(\frac{2}{0.031}\right) \ln \left(\frac{K_{c}}{R P T T}\right)-\frac{1}{3}\left(\frac{K_{a}}{R P T T^{2}}\right)$ $\pm \triangle D B P(\Delta T)$,

$S B P=D B P+K_{a}\left(\frac{1}{R P T T^{2}}\right) \pm \Delta S B P( \pm \Delta T)$,

where RPTT is the reflected pulse transit time, $M \mathrm{BP}$. is the mean blood pressure, $\triangle D B P(\Delta T)$ is the change in DBP due to temperature and $\triangle S B P( \pm \Delta T)$. is the changen DBP due to temperature. Equations (18), (19) (20) and (21) is used 
Fig. 27 Transient simulation of whole readout circuit with various gain

Fig. 28 The FFT simulation of whole readout circuit with input signal $1 \mathrm{~Hz}, 1 \mu \mathrm{A} \pm 0.02 \mu \mathrm{A}$ sine wave
Transient Analysis 'transient1-000_transient1': time $=(0 \mathrm{~s} s 10$

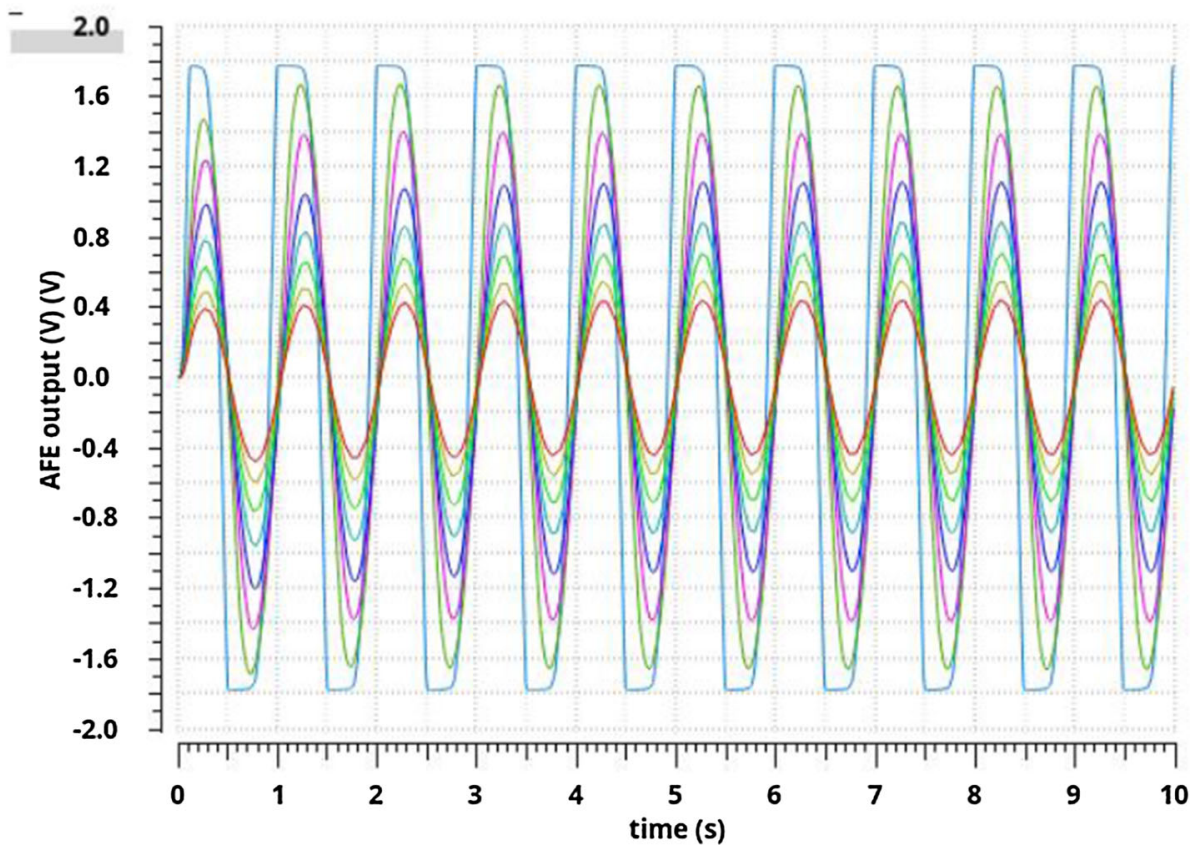

dB20(dft(value(value(value(value(value(value(value...g8" 1.8) 891024 "Rectangular" 1 "default" 1.01 $\because$

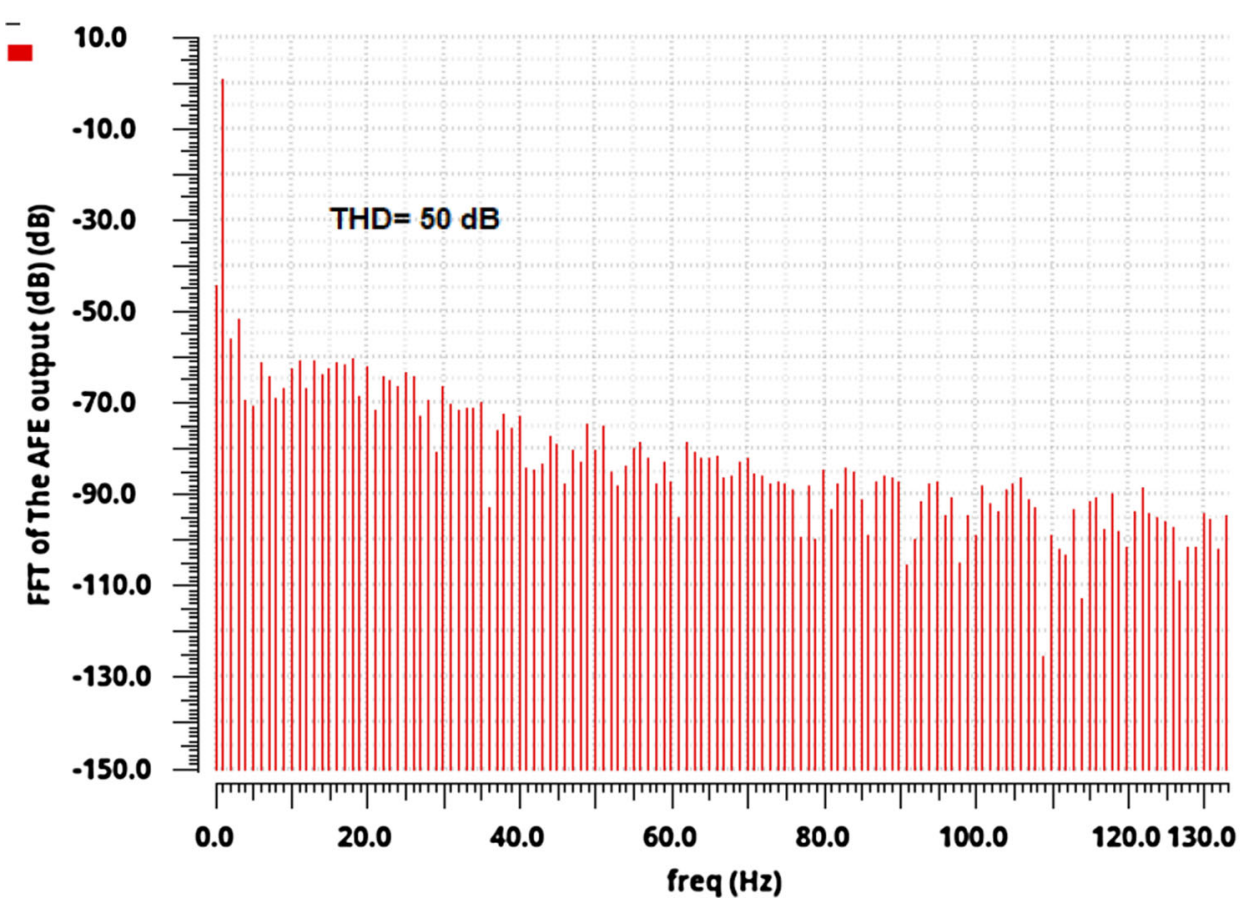

to calculate the $\mathrm{K}_{\mathrm{a}}, \mathrm{K}_{\mathrm{b}}$. \& $\mathrm{K}_{\mathrm{c}}$., by using the RPTT0 obtained from the first three cycles and using thitial vue SBP0 and DBP0. After calculating the fixed parameter $\mathrm{Ka}$, $\mathrm{Kb} \& \mathrm{Kc}$, real time BP is calculated by using Eq. (18), (19), (20), (21) and (22) and the RPTT (Kao et al. 2018). The measurement algorithm is shown in Fig. 23. According to this study the decrease in mean temperature below the skin temperature $\left(35^{\circ} \mathrm{C}\right.$, refer Fig. $24 \mathrm{a}$ and b) tends to $0.125 \mathrm{mmHg}$ and $0.063 \mathrm{mmHg}$ increase in SBP and DBP, respectively. Similarly, for every $1{ }^{\circ} \mathrm{C}$ increase in mean temperature above the mean ambient temperature $\left(35^{\circ} \mathrm{C}\right.$, refer to Fig. 24a and b) tends to $0.601 \mathrm{mmHg}$ and $0.121 \mathrm{mmHg}$ increase in SBP and DBP, respectively. 
Fig. 29 The wire-bond diagram

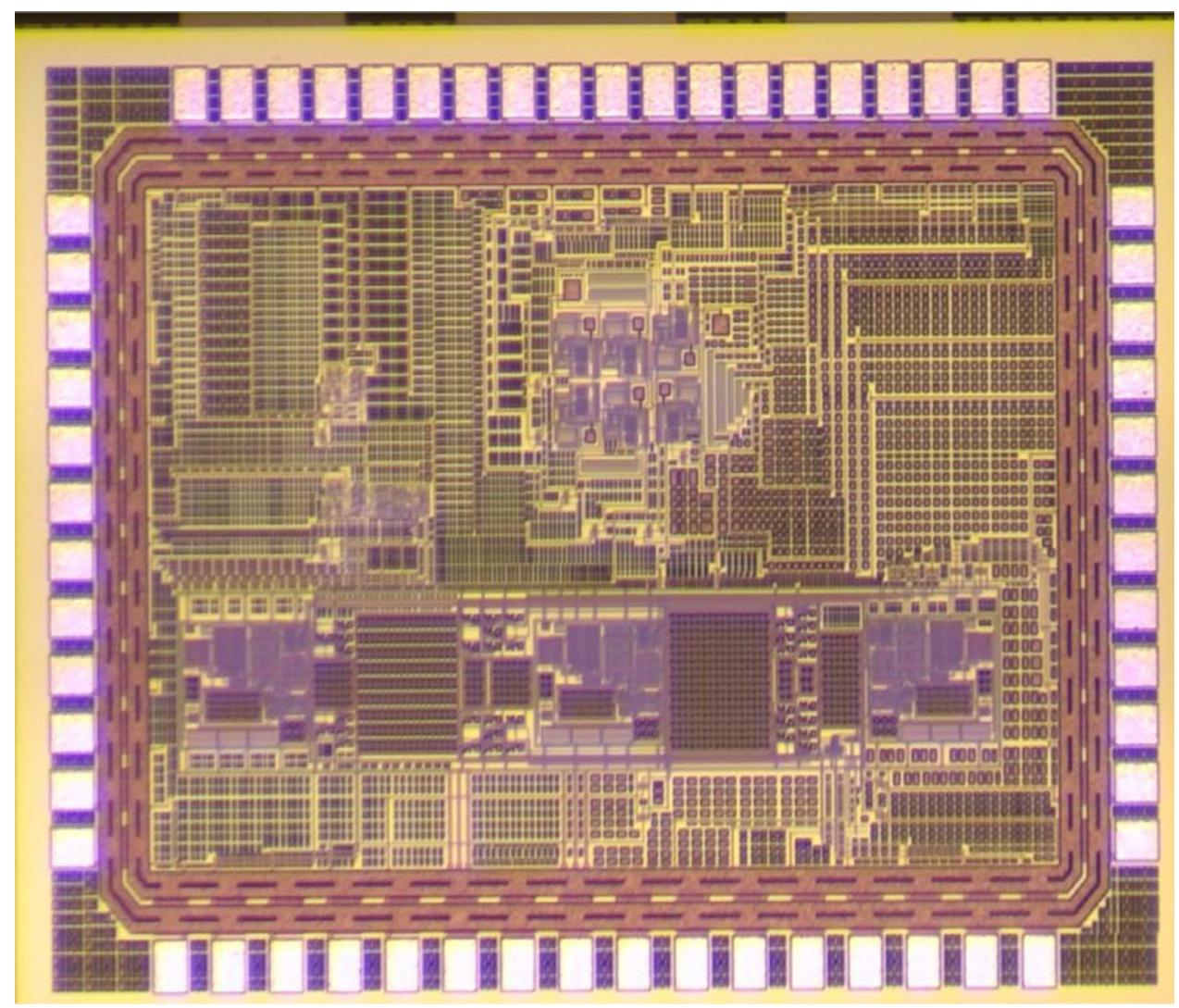

Fig. 30 AFE test environment
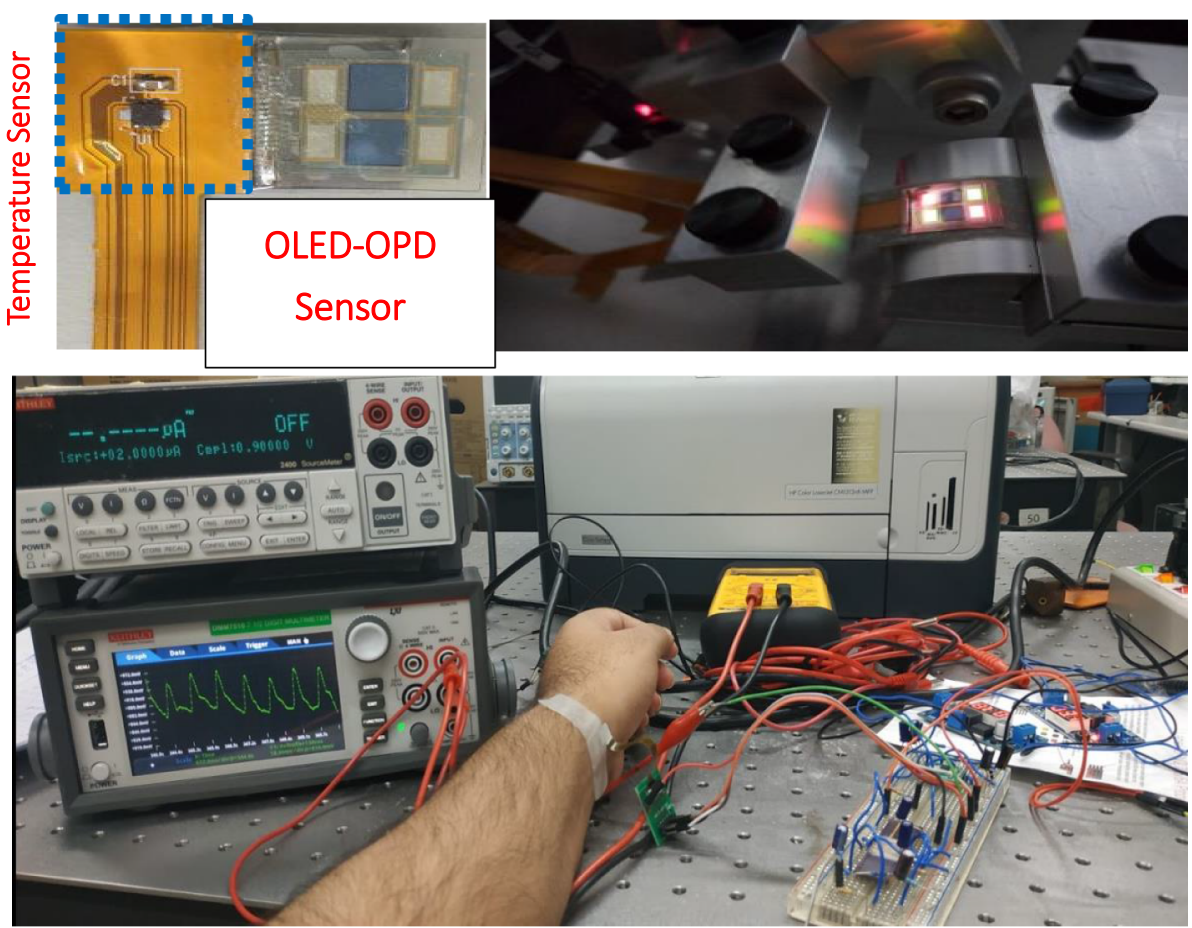


\section{Simulation and experimental results}

\subsection{Simulation and experimental results of the AFE}

With the OPD-PPG readout circuit and OLED-driver circuit designed successfully, an effort is devoted to the simulation and the experiment. The designed mixed signal readout circuit is first realized in a chip layout for the TSMC $0.18 \mu \mathrm{m}$ process. The power supply for the designed chip is $1.8 \mathrm{~V}$ readout. Simulations based on the chip layout are then carried out. AC and Transient analysis have been performed to validated the performance of the whole chip. Power supply rejection ratio (PSRR) with five corners of whole readout circuit is shown Fig. 25 which shows that the simulated PSRR is $120 \mathrm{~dB}$ at $60 \mathrm{~Hz}$. To get the accurate PPG signal, the noise and linearity are very important. The noise simulation of the designed readout circuit is shown in Fig. 26. The maximum rated input-referred noise is $202 \mathrm{pA}_{\mathrm{rms}}$. The transient simulation of

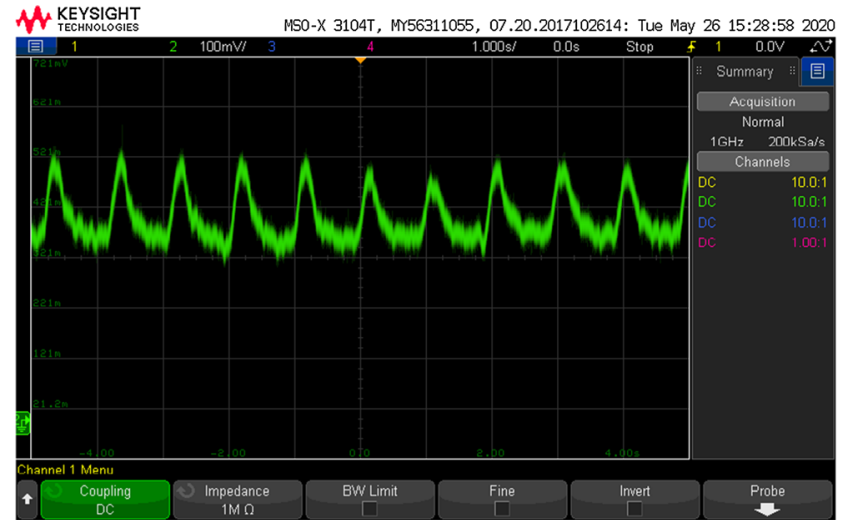

(a)

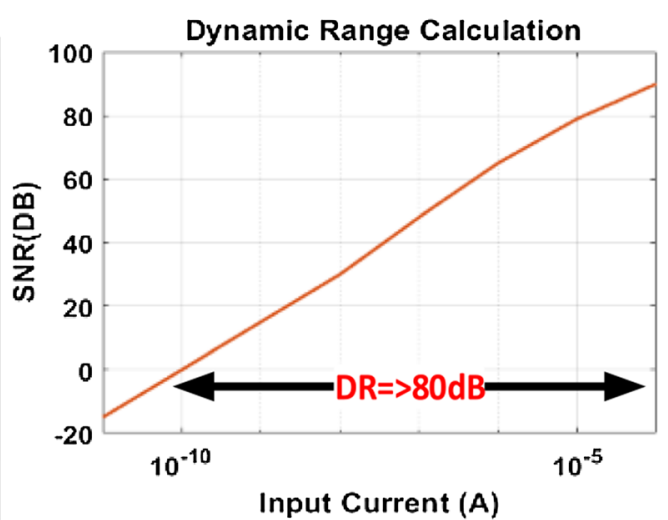

(b)

Fig. 31 a Output of the Readout system, b SNR of the readout system v.s. input current
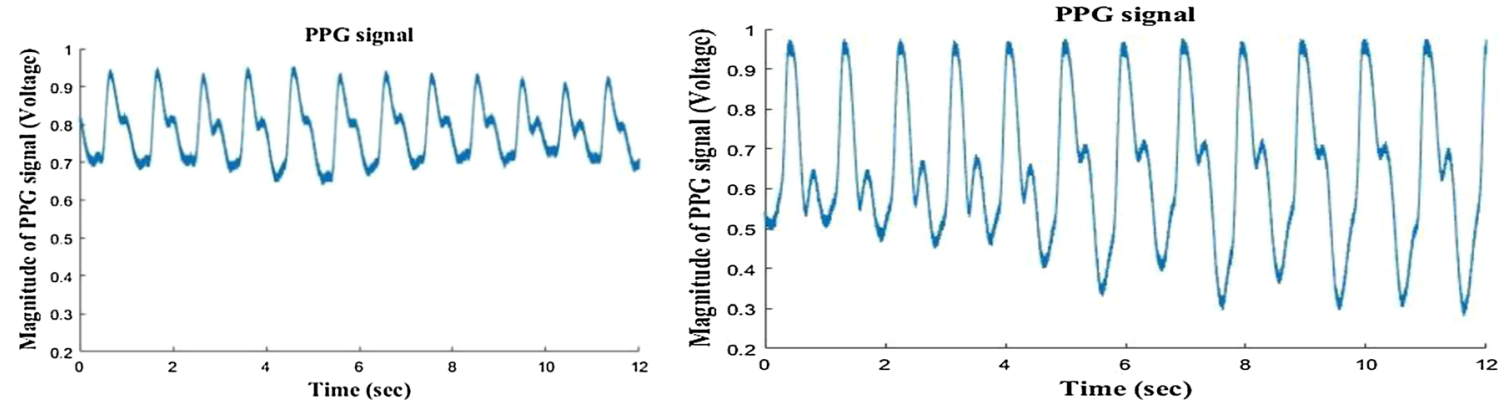

Fig. 32 The output of the readout circuit at different programmable TIA gain

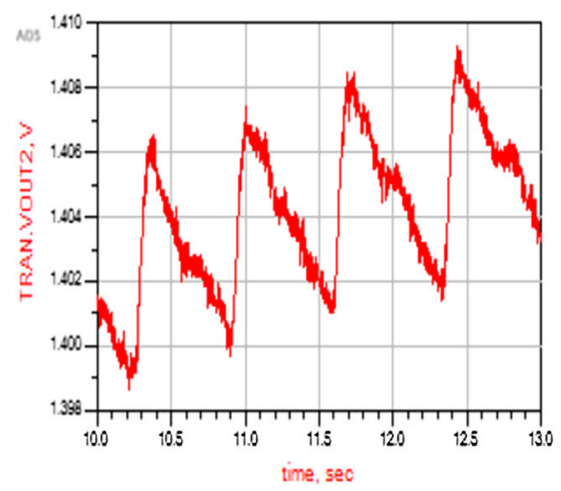

(a)

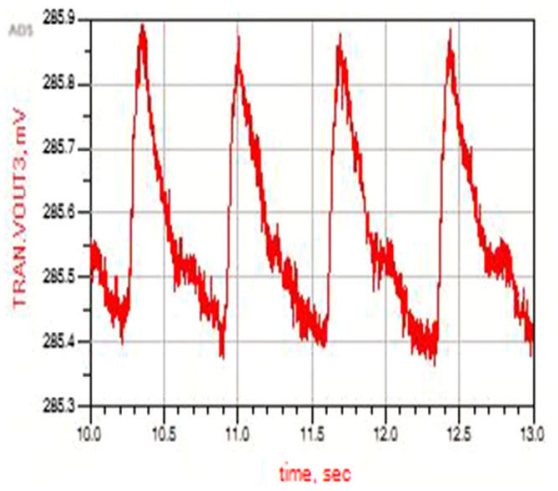

(b)

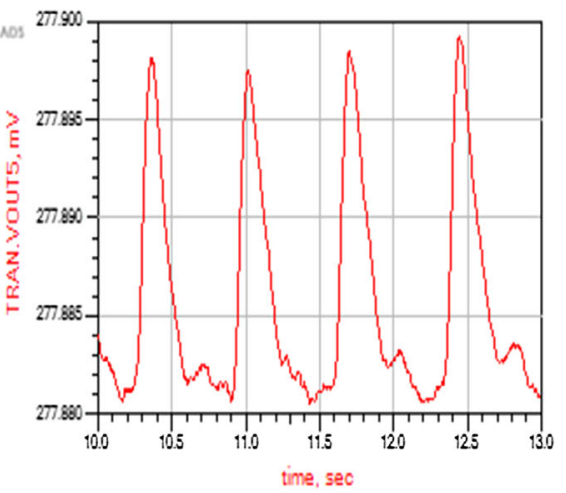

(c)

Fig. 33 Readout System output a without filtering, $\mathbf{b}$ with high pass filtering, $\mathbf{c}$ high pass filter followed by low pass filtering 


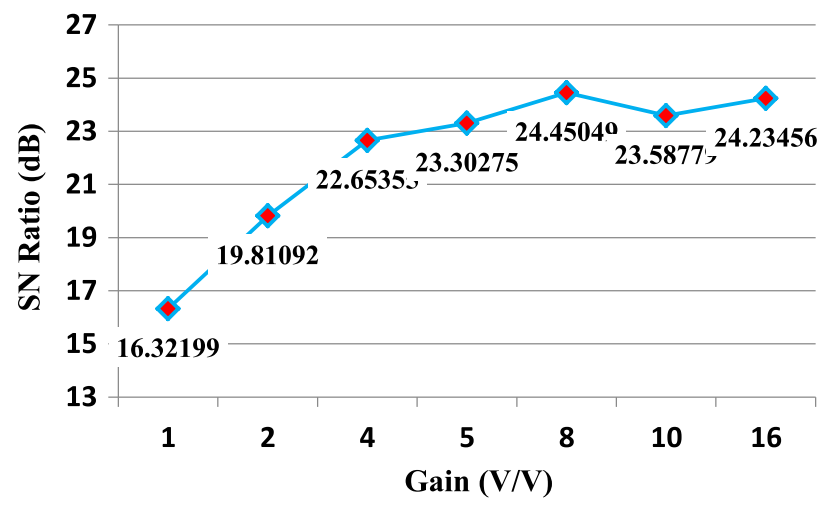

Fig. 34 SNR improvement with different PGA gain (@TIA gain $=$ minimum)

whole readout circuit with various gain is shown in Fig. 27. The simulation results show that the design readout circuit can achieve a dynamic gain of the $89 \mathrm{~dB}$. The output power spectrum density with input signal $1 \mathrm{~Hz}, 0.02 \mathrm{uA}$ sine wave simulation is shown in Fig. 28. The measured THD is $-51.8 \mathrm{~dB}$.

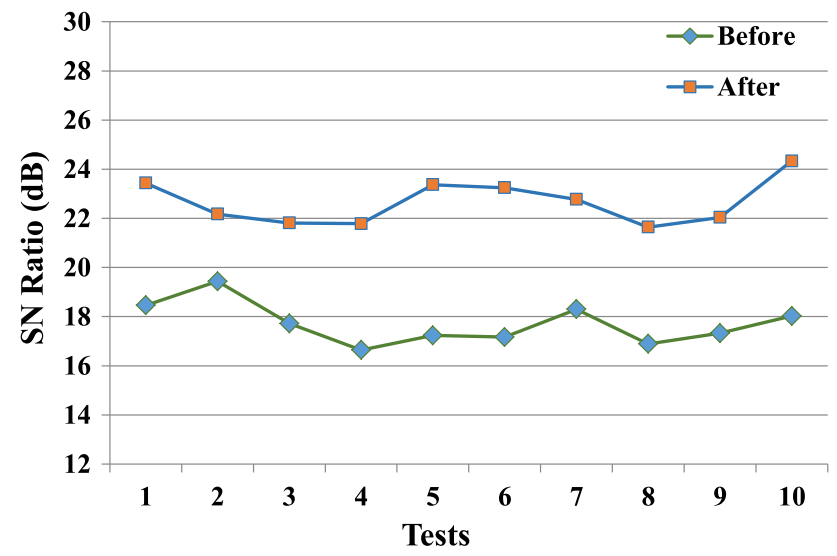

Fig. 36 The SNR of the PPG signals before and after the temperature compensation

The designed circuit has been next taped out by the TSMC $0.18 \mu \mathrm{m}$ process. The die photo is shown in Fig. 29, where the chip area is $1.2 \mathrm{~mm}^{2}$. It consists of a programmable TIA, a fourth-order $10 \mathrm{~Hz}$ low-pass filter, a second-order $0.1 \mathrm{~Hz}$ high-pass filter, a PGA and an OLED driver. The experiment setup is shown in Fig. 30. For the
Fig. 35 Received raw PPG signal a in normal condition, b temperature $<20^{\circ} \mathrm{C}$, c temperature $<20^{\circ} \mathrm{C}$ with the proposed digital compensation strategy
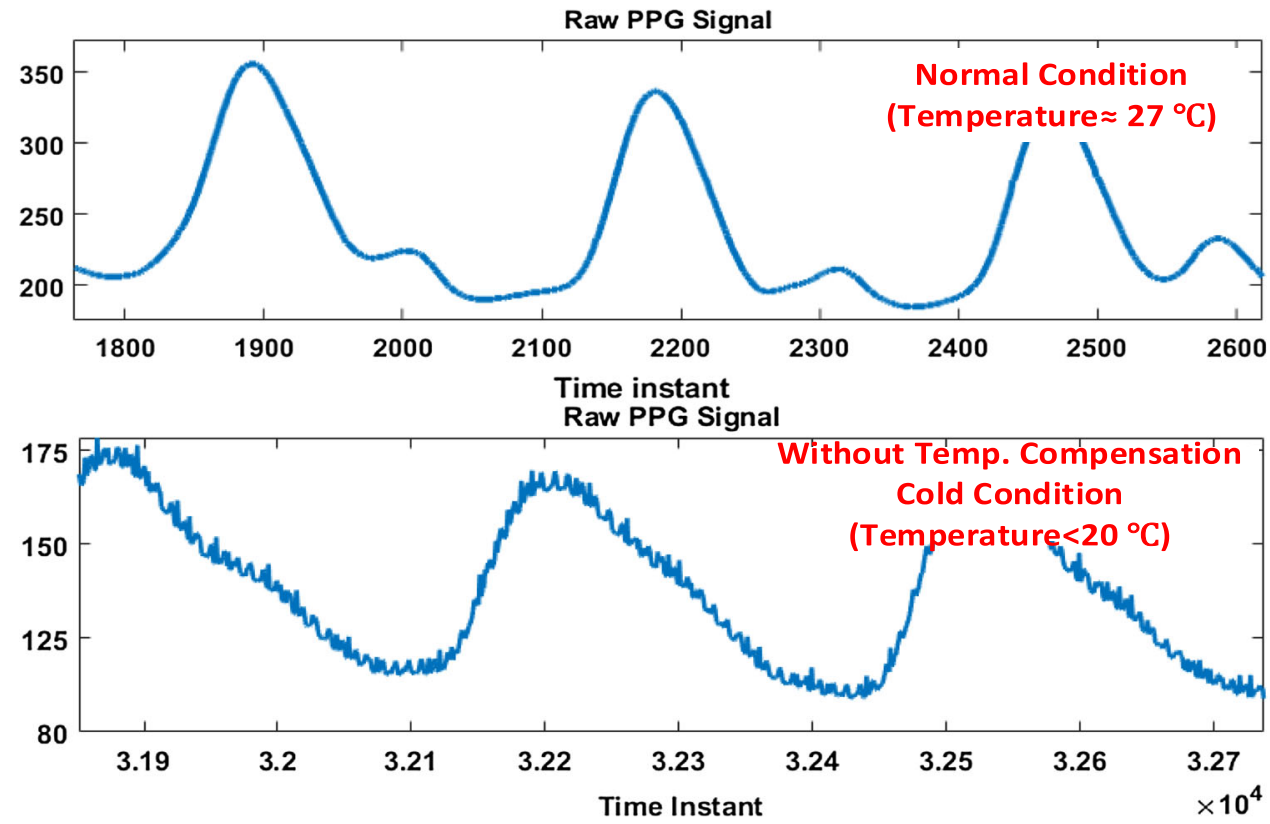

Raw PPG Signal

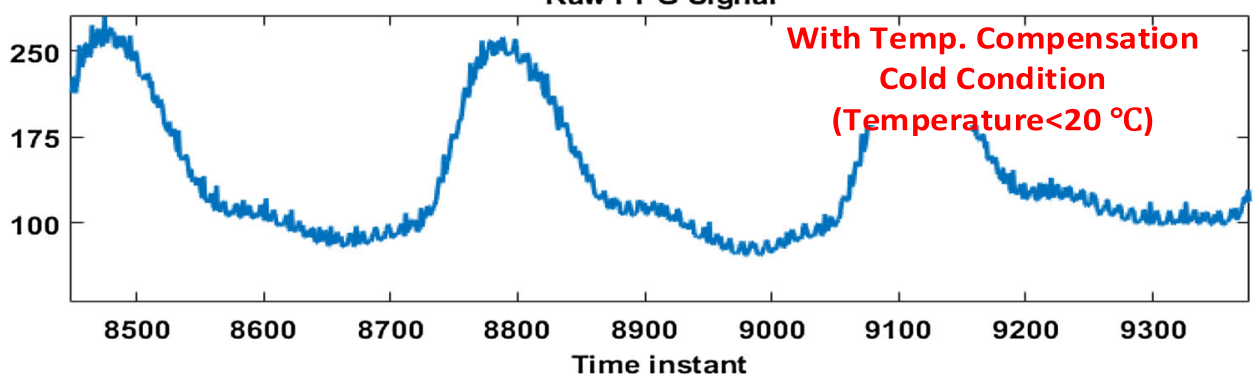


Fig. 37 The generated PWM Signal from the VCO for OLED intensity control

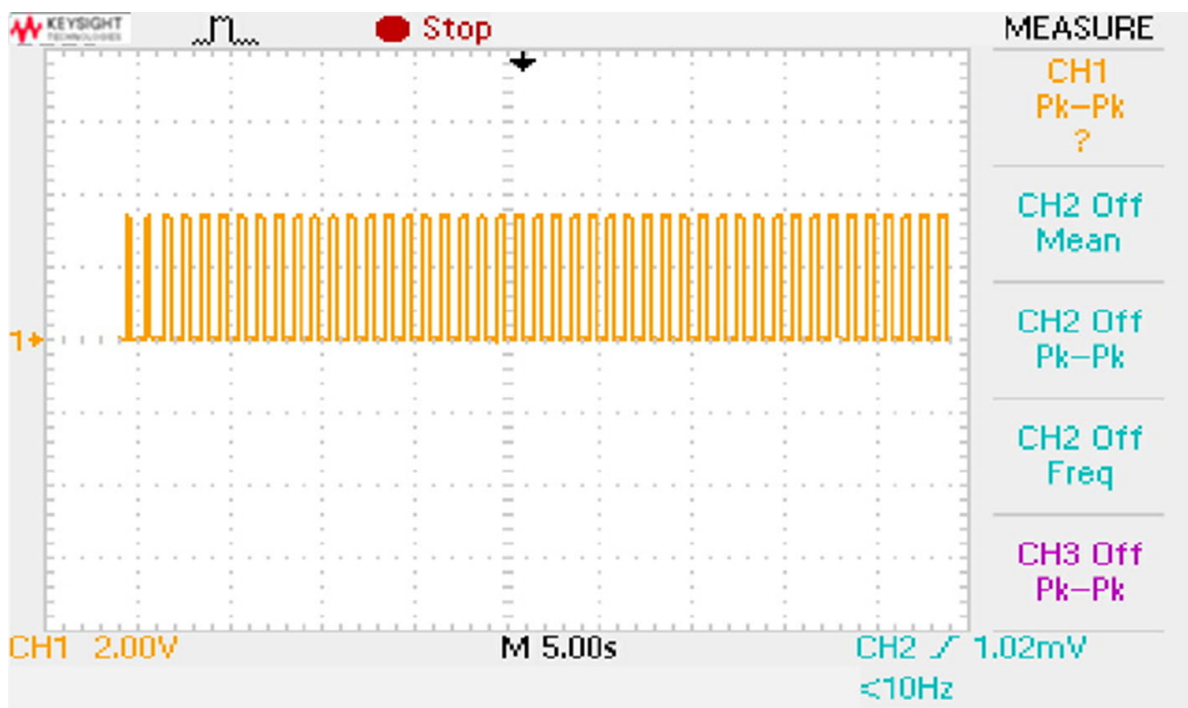

Table 1 Performance comparison of the PPG readout circuit

\begin{tabular}{|c|c|c|c|c|c|}
\hline Specification & This work & $\begin{array}{l}\text { Wong et al. } \\
\text { (2010) }\end{array}$ & $\begin{array}{l}\text { Sheng et al. } \\
(2016)\end{array}$ & $\begin{array}{l}\text { Pamula et al. } \\
\text { (2017) }\end{array}$ & Shu et al. (2020) \\
\hline $\begin{array}{l}\text { Supply } \\
\text { voltage }\end{array}$ & $1.8 \mathrm{~V}$ & $2.5 \mathrm{~V}$ & $3.3 \mathrm{~V}$ & $1.2 \mathrm{~V}$ & $1.8 / 2.8 \mathrm{~V}$ \\
\hline $\begin{array}{l}\text { Supply } \\
\text { current }\end{array}$ & $\begin{array}{l}16.8 \mu \mathrm{A} \text { (Readout) }+1.1 \mathrm{~mA} \text { (OLED } \\
\text { Driver) }+3.5 \mu \mathrm{A} \text { (temperature sensor }\end{array}$ & $58 \mu \mathrm{A}$ (readout) & $\begin{array}{l}455.2 \mu \mathrm{A} \\
\text { (readout) }\end{array}$ & $\begin{array}{l}143.3 \mu \mathrm{A} \\
\text { (readout) }\end{array}$ & $40 \mu \mathrm{A}$ (readout) \\
\hline $\begin{array}{l}\text { Program. } \\
\text { TIA }\end{array}$ & Yes & No & No & Yes & Yes \\
\hline $\begin{array}{l}\text { Sensing } \\
\text { Current }\end{array}$ & $20 \mathrm{nA}-100 \mu \mathrm{A}$ & $400 \mathrm{nA}$ & NA & NA & $\approx 200 \mu \mathrm{A}$ \\
\hline Gain $(\mathrm{dB})$ & $135-155$ & 63.5 & 60 & - & $80 @ 6 \mu \mathrm{A}$ \\
\hline $\begin{array}{l}\text { HPF } 3 \mathrm{~dB} \\
\text { Cutoff }\end{array}$ & 0.2 & $0.5-110$ & 0.2 & 0.5 & 0.5 \\
\hline $\begin{array}{l}\text { LPF } 3 \mathrm{~dB} \\
\text { Cutoff }\end{array}$ & $10 \mathrm{~Hz}$ & - & 20 & 3.5 & $20 \mathrm{~Hz}$ \\
\hline $\begin{array}{l}\text { Input } \\
\text { referred } \\
\text { noise }\end{array}$ & $60.2 \mathrm{pA} / \mathrm{Hz}^{1 / 2}$ & $\begin{array}{l}40.8 \mathrm{nA} \\
\text { (integrated } \\
\text { noise) }\end{array}$ & 1.8 & 0.486 Arms & $0.7 \mu \mathrm{Vrms}$ \\
\hline Power & $\begin{array}{l}30.24 \mu \mathrm{W} \text { (readout) + OLED Driver } \\
(1.62 \mathrm{~mW} @ 100 \% \text { duty cycle })\end{array}$ & $\begin{array}{l}145 \mu \mathrm{W} \text { without } \\
\text { LED driver }\end{array}$ & $\begin{array}{l}1502 \mu \mathrm{W} \text { without } \\
\text { LED driver }\end{array}$ & $\begin{array}{l}172 \mu \mathrm{W} \text { without } \\
\text { LED driver }\end{array}$ & $\begin{array}{l}70 \mu \mathrm{W} \text { without } \\
\text { LED driver }\end{array}$ \\
\hline Chip size & $2.507 \mathrm{~mm}^{2}$ & $0.67 \mathrm{~mm}^{2}$ & $0.68 \mathrm{~mm}^{2}$ & $10 \mathrm{~mm}^{2}$ & $4.5 \mathrm{~mm}^{2}$ \\
\hline Process & $0.18 \mu \mathrm{m}$ & $0.35 \mu \mathrm{m}$ & $0.18 \mu \mathrm{m}$ & $0.18 \mu \mathrm{m}$ & $0.055 \mu \mathrm{m}$ \\
\hline
\end{tabular}

experiment OLED-OPD senor patch is attached to AFE. Then the non-invasive PPG readout is applied to the wrist artery of the subject. The acquired PPG signal is shown in Fig. 31a. According to the Fig. 31b The measured dynamic range $\left(\mathrm{I}_{\text {Minimum }} / \mathrm{I}_{\text {Maximum }}\right)$ of the readout circuit is $89 \mathrm{~dB}$. Since the maximum PD current is $11 \mathrm{uA}$, therefore the measured SNR at the 11uA input current is 65 dB (@ PGA gain $=0 \mathrm{~dB})$. The output of the readout circuit at different TIA gain is shown in Fig. 32. Figure 33a shows the output of the readout circuit without filtering. Figure $33 \mathrm{~b}$ shows the output with the 2 nd order high pass filter and Fig. 33c shows the output with 2 nd order high pass filter and 4th order low pass filter. This shows that the DC drift and high frequency noise is significantly reduced due to the 
Table 2 Characteristics of the subjects

\begin{tabular}{ll}
\hline Total number of subject & 40 subject \\
Number of males subject & 36 \\
Number of female subject & 4 \\
Subject with fever (body temp $\left.>37^{\circ} \mathrm{C}\right)$ & 1 \\
Age range & $(20-48)$ years \\
SBP range & $(91-163) \mathrm{mmHg}$ \\
DBP range & $(53-103) \mathrm{mmHg}$ \\
Subjects with SBP above $130 \mathrm{mmHg}$ & 5 \\
\hline
\end{tabular}

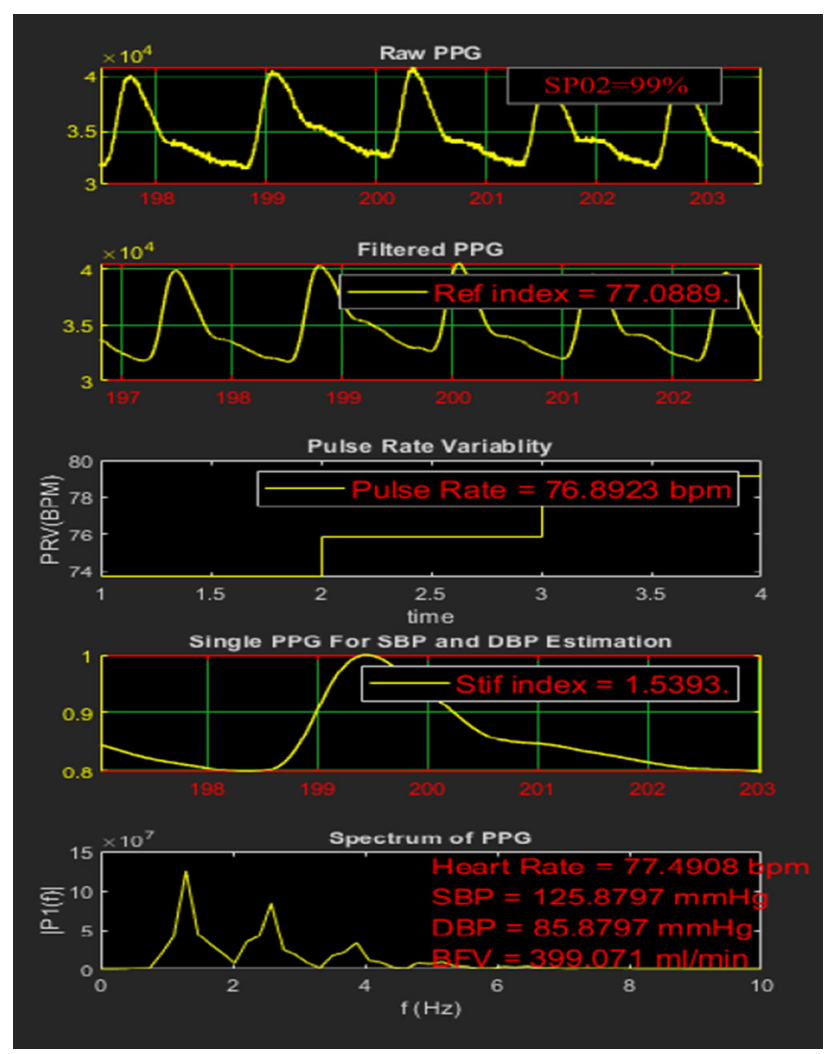

Fig. 38 Design graphics-user-interface (GUI) for the different bio signs detection

$0.1-10 \mathrm{~Hz}$ band pass filter. The SNR improvement with the PGA (@TIA Gain = minimum) is shown in Fig. 34, which shows that the SNR is improved by the $8 \mathrm{~dB}$. The integrated temperature sensor is used to measure the skin temperature in parallel with the PPG signal. This data has been used for the temperature compensation to improve the PPG quality at the low ambient temperature. Figure 35 (a) shows the received PPG signal in the normal environment (temperature $=27^{\circ} \mathrm{C}$ ). Figure 35b shows the received PPG signal in the cold environment (temperature $<20{ }^{\circ} \mathrm{C}$ ). While Fig. $35 \mathrm{c}$ shows the received PPG signal with the temperature compensation strategy as discuss in Sect. 2.5 and shown in Figs. 20, 21. According to the Fig. 35c, it can be seen that the quality of the PPG signal is improved. Point to be noted that the TIA gain is kept constant in all the three aforementioned cases (Fig. 35a-c. Therefore, the PPG signal quality in terms of the SNR, before and after the temperature compensation is shown in Fig. 36, which shows that the SNR is improved by the $6 \mathrm{~dB}$ approximately. The generated $400 \mathrm{~Hz}$ PWM signal from the VCO for OLED intensity control is shown in Fig. 37. The comparison of the designed readout circuit with previous state-of-arts are compiled in Table 1. The comparison results show that the designed circuit perform better in terms of the of the total power consumption, DC drifting, noise etc.

\subsection{Experimental results for the $\mathrm{HR}, \mathrm{S}_{\mathrm{p}} \mathrm{O}_{2}$ and $\mathrm{BP}$ Detection}

The flexible sensor is directly attached to the skin hence there will be no relative motion between the sensing spot and the sensor. Therefore, the design OLED-OPD flexible patch with the low power AFE helps to alleviate the impact of the motion artifact, thus enhance the long time continuous and accurate monitoring of the Spo2, HR and BP. The non-invasive PPG acquisition system is applied to the wrist artery of the 40 healthy subjects for sensing the pulsation of the blood vessel under the different Air conditioner environment (cold $<20{ }^{\circ} \mathrm{C}$ to hot $32{ }^{\circ} \mathrm{C}+1$ patient with fever whom body temperature was $\approx 40{ }^{\circ} \mathrm{C}$ ). The characteristics of the subjects participated in the research are compiled in Table 2. For the ground truth, an OMRON (HR and BP) and a Rossmax $\left(\mathrm{S}_{\mathrm{p}} \mathrm{O}_{2}\right)$ device has been used as the gold standard. The designed graphics user interface is shown in Fig. 38. According to this study for every $1{ }^{\circ} \mathrm{C}$ decrease in mean temperature below the mean ambient temperature $\left(35^{\circ} \mathrm{C}\right.$, refer to Figs. $21 \mathrm{c}$ and $24 \mathrm{a}$ and b) tends to $0.06 \mathrm{bps}$, $0.125 \mathrm{mmHg}$ and $0.063 \mathrm{mmHg}$ increase in HR, SBP and DBP, respectively. Similarly, for every $1{ }^{\circ} \mathrm{C}$ increase in mean ambient temperature above the skin temperature $\left(35^{\circ} \mathrm{C}\right.$, refer to Figs. $21 \mathrm{c}$ and $24 \mathrm{a}$ and b) tends to $0.13 \mathrm{bps}$, $0.601 \mathrm{mmHg}$ and $0.121 \mathrm{mmHg}$ increase in HR, SBP and DBP, respectively. However, the individual body characteristics could shows the different characteristics. The estimated heart rate vs ground truth is shown in Fig. 39a, which shows that the correlation factor is $\mathrm{R}^{2}=91 \%$. In addition, the error in terms of mean error $\pm 1.96^{*}$ standard deviation is shown in Fig. 39b. The measurement results show that the heart rate accuracy and standard error are $96 \%$, and $-0.022 \pm 2.589 \mathrm{bpm}$, respectively. The measured $\mathrm{S}_{\mathrm{p}} \mathrm{O}_{2}$ results on the same subject at 3 times in a day is shown in Fig. 40. According to the experiment, it is observed that the impact of the temperature on the $\mathrm{S}_{\mathrm{p}} \mathrm{O}_{2}$ is less than the $1 \%$. The estimated $\mathrm{S}_{\mathrm{p}} \mathrm{O}_{2}$ measurement error is 
Fig. 39 a The estimated heart rate vs ground truth, and $\mathbf{b}$ the error plot

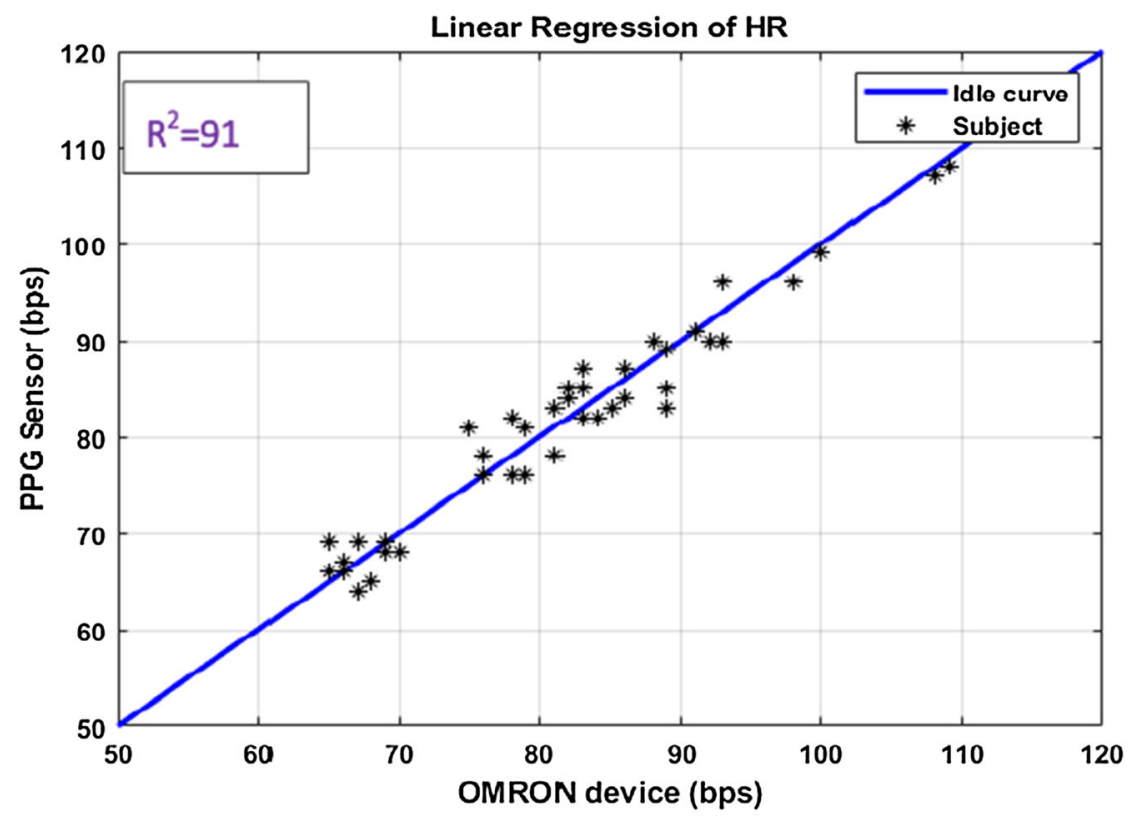

(a)

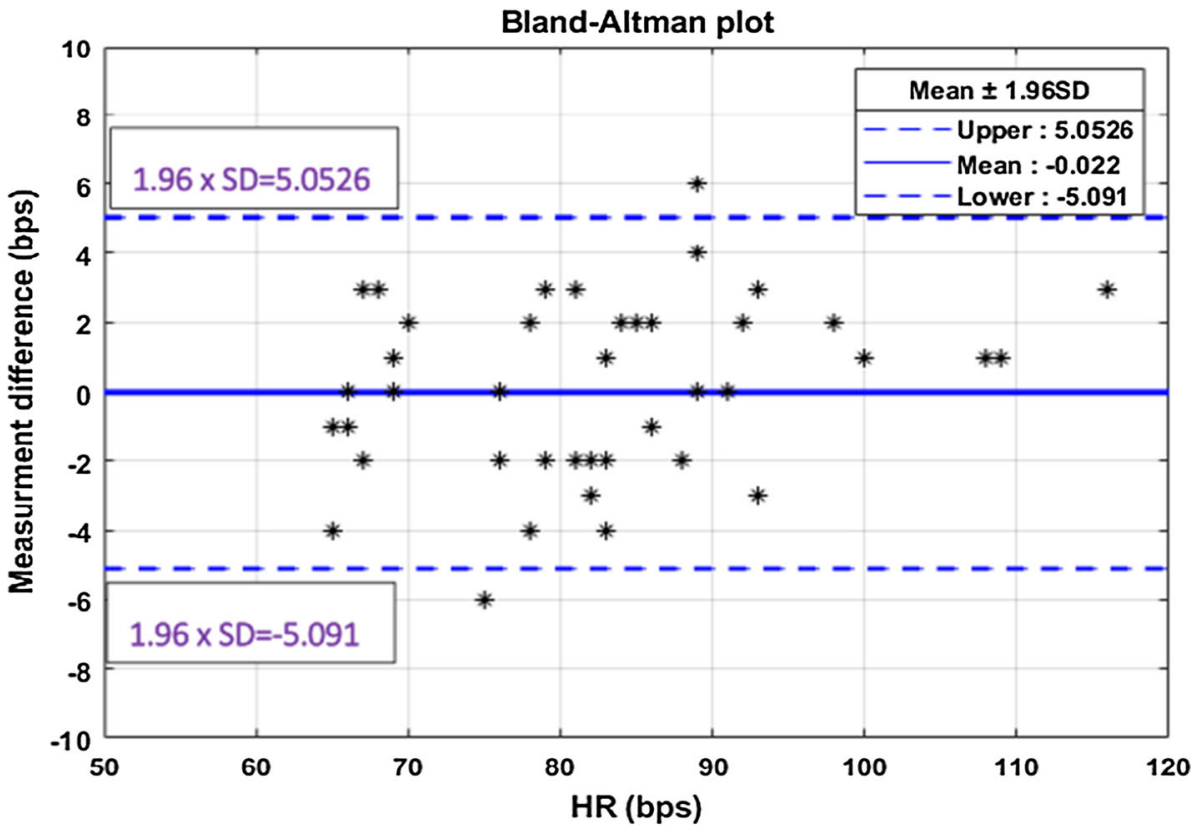

(b) shown in Fig. 41, which shows that mean absolute percentage error (MAPE) is less than 5\%. Figure 42a and b shows the Bland-Altman error plot of estimating SBP and DBP. The SBP measurement error is $-0.29 \pm 5.19 \mathrm{mmHg}$, while the error in DBP measurement is $-0.5 \pm 1.91 \mathrm{mmHg}$. Similarly, Fig. 43a shows the correlation between the measured SBP and the standard OMRON device. Also, Fig. 43b shows the correlation between the measured DBP and the standard OMRON device. The measure SBP and DBP correlation are $89 \%$ and $96 \%$ respectively. According to the Advancement of Medical Instrumentation (AAMI) and British Hypertension Society (BHS) standard, the performance of the device is in Grade A. 


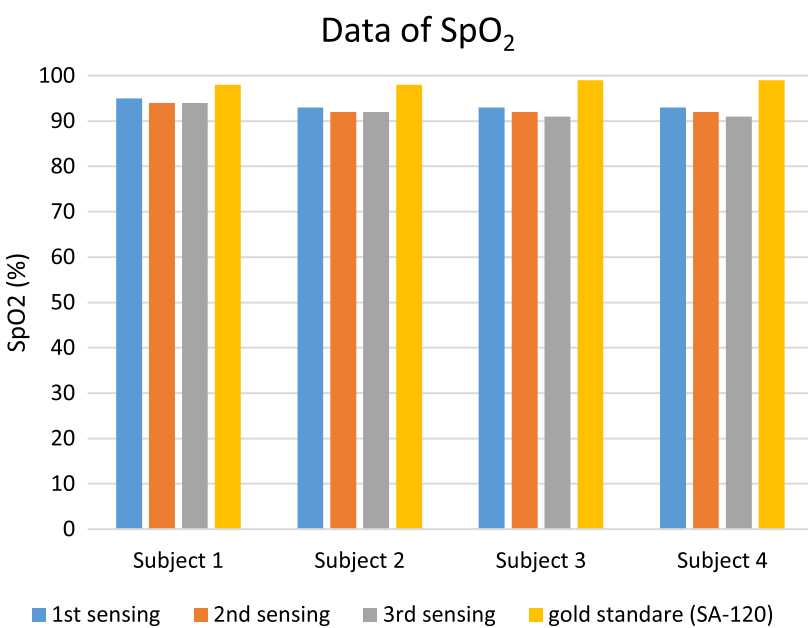

Fig. 40 Blood oxygen saturation measurement results

\section{Conclusion}

This study presents a temperature sensor assisted time-interleave, wide dynamic range, low power and low DC drift photoplethysmography (PPG) signal acquisition system to obtain the accurate measurement of the heart rate, blood oxygen and blood pressure. With the standard $1.8 \mathrm{~V}$, the experimental result shows that the measured current sensing range is $20 \mathrm{nA}-100 \mathrm{uA}$. The dynamic range of the design readout circuit is $80 \mathrm{~dB}$. The estimated signal to noise ratio is $60 \mathrm{~dB} @ 1 \mathrm{uA}$. The measured input referred noise is $60.2 \mathrm{pA} / \mathrm{Hz}^{1 / 2}$. The total power consumption of the design chip is $31.32 \mu \mathrm{W}$ (readout) $+1.62 \mathrm{~mW}$ (OLED@100\% duty cycle. This study concludes that for every $1{ }^{\circ} \mathrm{C}$ decrease in mean ambient temperature tends to $0.06 \mathrm{bps}, 0.125 \mathrm{mmHg}$ and $0.063 \mathrm{mmHg}$ increase in HR, SBP and DBP, respectively. Similarly, for every $1^{\circ} \mathrm{C}$ increase in mean ambient temperature tends to $0.13 \mathrm{bps}$, $0.601 \mathrm{mmHg}$ and $0.121 \mathrm{mmHg}$ increase in HR, SBP and DBP, respectively. However, the individual body characteristics could shows the different characteristics. The measured accuracy and standard error for the HR estimation are $96 \%$, and $-0.022 \pm 2.589 \mathrm{bpm}$, respectively. The $\mathrm{S}_{\mathrm{p}} \mathrm{O}_{2}$ measurement results shows that the means absolute percentage error is less than 5\%. The resultant errors for the SBP and DBP measurement are $-0.29 \pm 5.19 \mathrm{mmHg}$ and $-0.5 \pm 1.91 \mathrm{mmHg}$, respectively. According to the Advancement of Medical Instrumentation (AAMI) and British Hypertension Society (BHS) standard, the measured error of Grade "A" devices must be less than $8 \mathrm{mmHg}$. Based on the above BP measurement from the designed device shows that the performance of the device is in Grade A.
Fig. 41 Standard Error plot for the blood oxygen saturation measurement

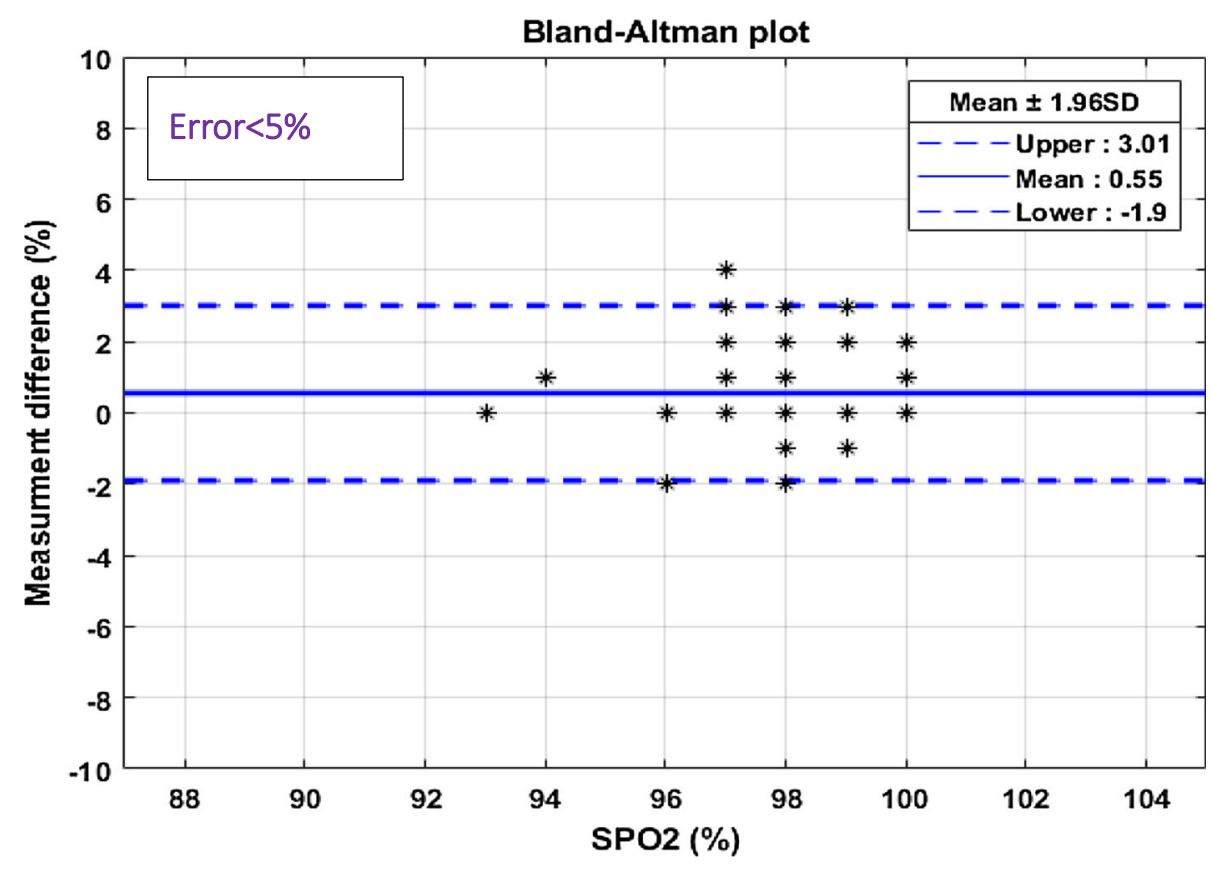


Fig. 42 Distribution range of measured BP error by 40 subjects at a SBP; b DBP

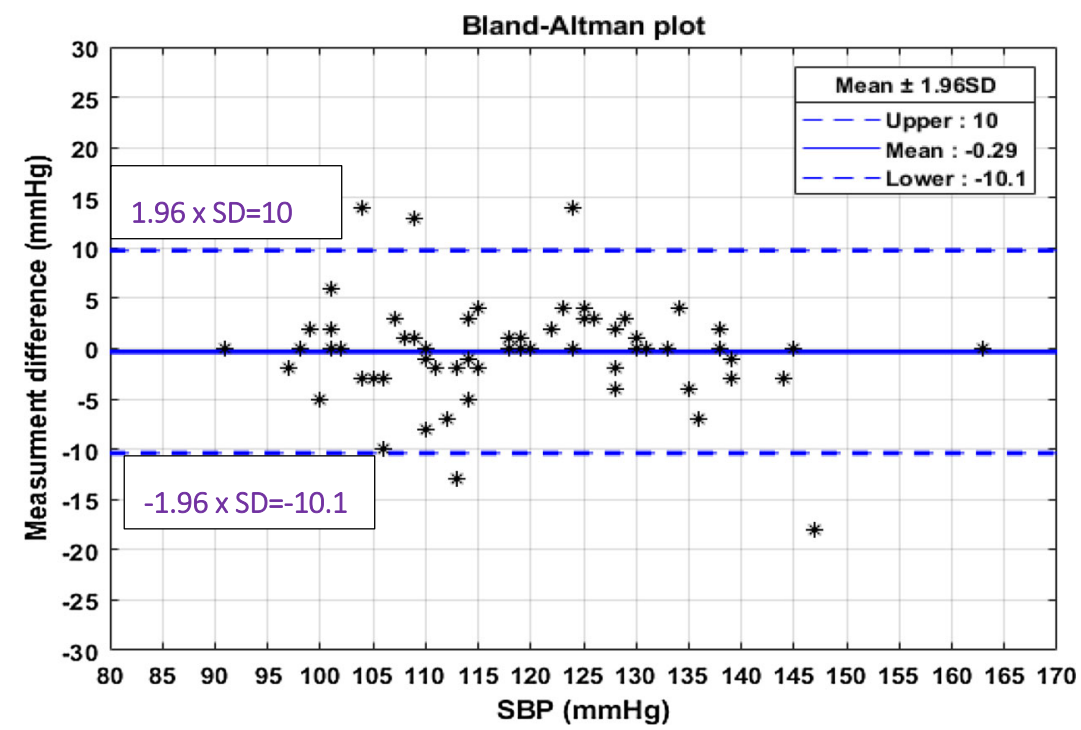

(a)

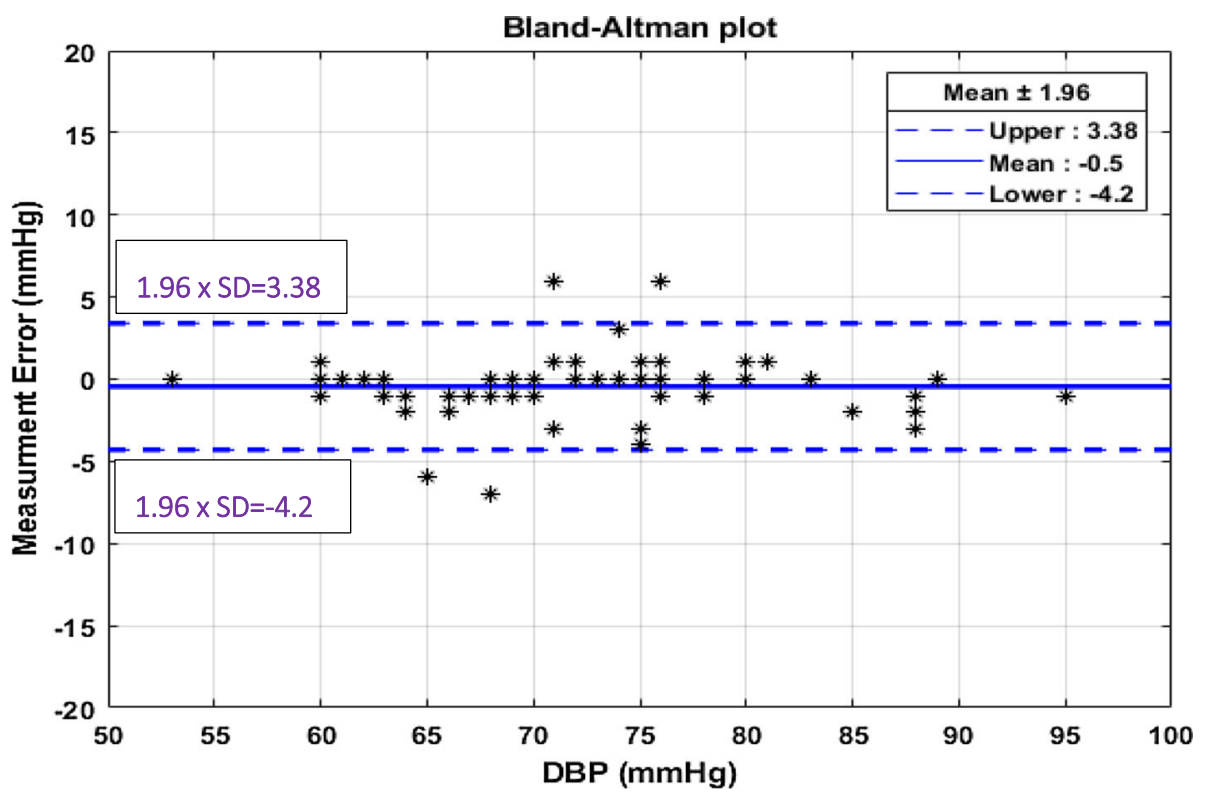

(b) 
Fig. 43 Correlation plot of $\mathrm{BP}$ by 40 subjects at a SBP; b DBP

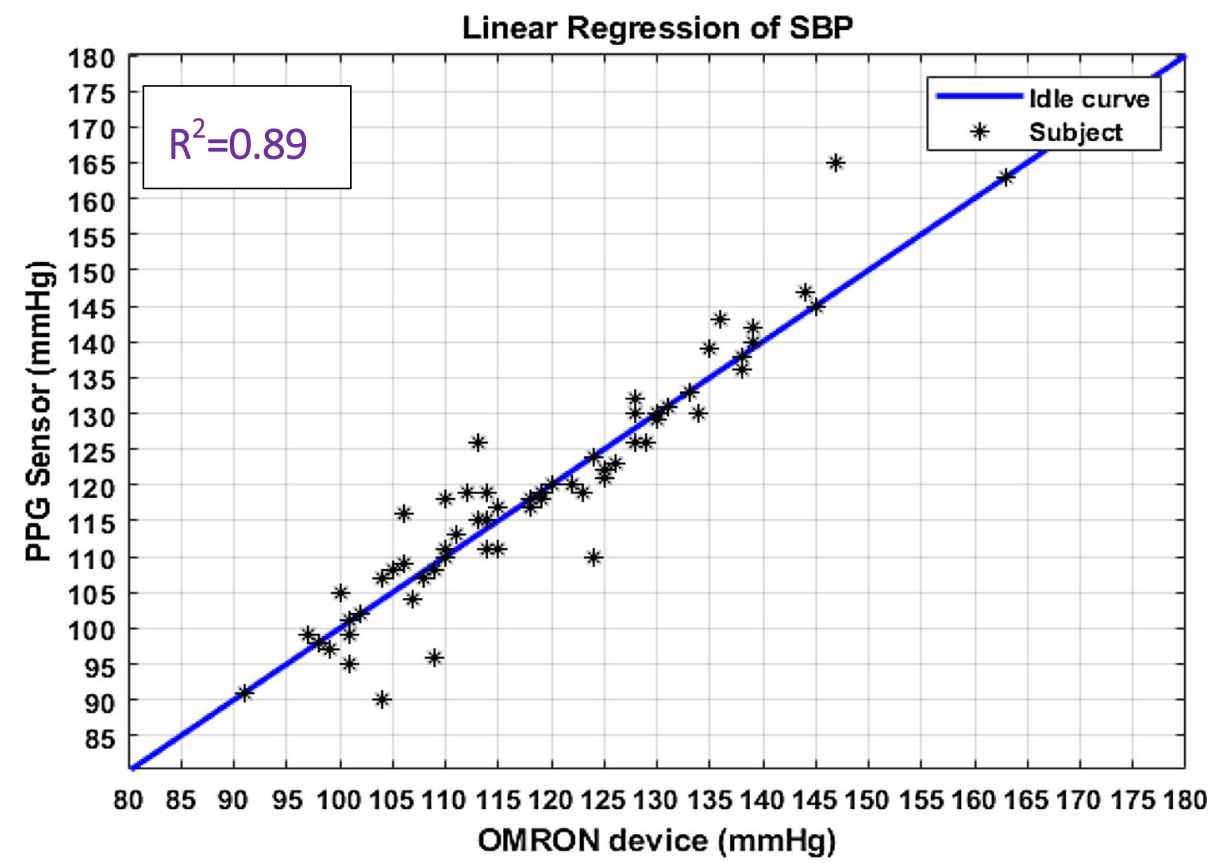

(a)

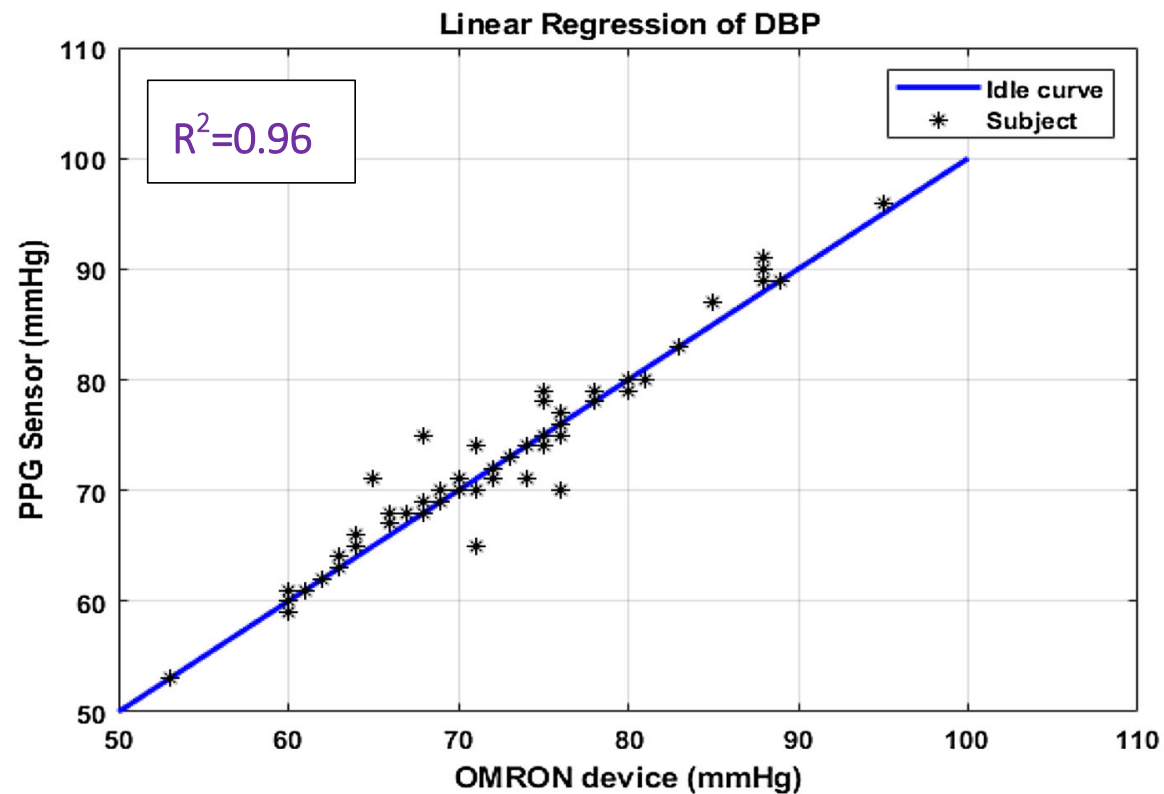

(b)
Acknowledgements The authors appreciate the supports from Taiwan semiconductor research institute (TSRI).This study is supported by Ministry of Science and Technology, Taiwan grant No. MOST 108-2823-8-009 -002 -, 109-2622-8-009-018 -TE1, and 109-2221-E009-163 -. This work was financially supported by the "Center for Intelligent Drug Systems and Smart Bio-devices (IDS2B)" from The Featured Areas Research Center Program within the framework of the Higher Education Sprout Project by the Ministry of Education (MOE) in Taiwan. It was also supported in part by Hsinchu Science Park Bureau, MOST grant No. 108A31B.

\section{References}

Ahmad S, Chen S, Soueidan K, Batkin I, Bolic M, Dajani H, Groza V (2012) Electrocardiogram-assisted blood pressure estimation. IEEE Trans Biomed Eng 59(3):608-618

Bonomi, AG, Schipper F, Eerikäinen LM, Margarito J, Aarts RM, Babaeizadeh S, Morree HM, Dekker LG (2016) Atrial fibrillation detection using photo-plethysmography and acceleration data at the wrist. In: 2016 Computing in Cardiology Conference (CinC) (2016), pp 277-280

Bramwell JC, Hill AV (1992) The velocity of the pulse wave in man. Proc R Soc Lond Biol Character 93(652):298-306 
Cohen Z, Haxha S (2017) Optical-based sensor prototype for continuous monitoring of the blood pressure. IEEE Sens J 17(13):4258-4268

Forouzanfar M, Ahmad S, Batkin I, Dajani HR, Groza VZ, Bolic M (2013) Coefficient-free blood pressure estimation based on pulse transit time-cuff pressure dependence. IEEE Trans Biomed Eng 60(7):1814-1824

Guglielmi E et al (2020) High-value tunable pseudo-resistors design. IEEE J Solid-State Circuits. https://doi.org/10.1109/JSSC.2020. 2973639

Higgins C, Little (2014) Oxygen saturation-better measured than calculated May 2014. Published in https://acutecaretesting.org/

Huang S-C, Hung P-H, Hong C-H, Wang H-M (2014) A new image blood pressure sensor based on PPG, RRT, BPTT, and harmonic balancing. IEEE Sens J 14(10):3685-3692

Kao YH, Chao P-CP, Wey CL (2018) Towards maximizing the sensing accuracy of an cuffless, optical blood pressure sensor using a high-order front-end filter. Microsyst Technol 24:4621

Kao YH, Chao P-CP, Wey CL (2019) Design and validation of a new PPG module to acquire high-quality physiological signals for high-accuracy biomedical sensing. IEEE J Sel Top Quantum Electron 25(1):1-10 (Art no. 69000210)

Khalil O, Yeh S, Lowery Michael G, Wu X, Hanna C, Kantor S, Jeng T, Kanger JS, Bolt R, de Mul FD (2003) Temperature modulation of the visible and near infrared absorption and scattering coefficients of human skin. J Biomed Optics 8(2):191-205

Khan M, Pretty CG, Amies AC, Elliott R, Shaw GM, Chase JG (2015) Investigating the effects of temperature on photoplethysmography. IFAC -Papers OnLine,Volume 48, Issue 20, 2015, pp 360-365, ISSN 2405-8963

Khan Y, Han D, Ting J, Ahmed M, Nagisetty R, Arias AC (2019) Organic multi-channel optoelectronic sensors for wearable health monitoring. IEEE Access 7:128114-128124. https://doi. org/10.1109/Access.2019.2939798

Lee Y et al (2017) Sticker-type hybrid photoplethysmogram monitoring system integrating CMOS IC with organic optical sensors. IEEE J Emerg Sel Top Circuits Syst 7(1):50-59. https://doi.org/ 10.1109/JETCAS.2016.2630301.1109/TBCAS.2019.2945114

Lee SY, Cheng CJ (2009) Systematic design and modeling of a OTAC filter for portable ECG detection. IEEE Trans Biomed Circuits Syst 3(1):53-64

Lin Q et al. (2019) A $196 \mu \mathrm{W}$, reconfigurable light-to-digital converter with $119 \mathrm{~dB}$ dynamic range, for wearable PPG/NIRS sensors. In: 2019 Symposium on VLSI Circuits (2019): C58-C59

Lochner C, Khan Y, Pierre A et al (2014) All-organic optoelectronic sensor for pulse oximetry. Nat Commun 5:5745. https://doi.org/ 10.1038/ncomms6745

Madaniyazi L, Zhou Y, Li S et al (2016) Outdoor temperature, heart rate and blood pressure in chinese adults: effect modification by individual characteristics. Sci Rep 6:21003. https://doi.org/10. 1038/srep21003

Marefat $\mathrm{F}$ et al (2020) A 1-V $8.1 \mathrm{uW}$ PPG-recording front-end with > 92-dB DR using light-to-digital conversion with signal-aware DC subtraction and ambient light removal. IEEE Solid-State Circuits Lett 3:17-20

Orozco LM (2013) Programmable-gain transimpedance amplifiers maximize dynamic range in spectroscopy systems. Analog Dev

Pamula VR, Valero-Sarmiento JM, Yan L, Bozkurt A, Van Hoof C, Van Helleputte N, Verhelst M (2017) A 172 uW compressively sampled photoplethysmographic (PPG) readout ASIC with heart rate estimation directly from compressively sampled data. IEEE Trans Biomed Circuits Syst 11(3):487-496

Pandey RK, Pribadi EF, Chao P-C (2019) A new adaptive readout system for a new OLED OPD flexible patch PPG sensor. In: 2019 IEEE Sensors, Montreal, QC, Canada, 2019, pp 1-4, doi: https://doi.org/10.1109/sensors43011.2019.8956825

Prahl S (1999) http://omlc.Ogi.Edu/spectra/hemoglobin

Pribadi EF, Pandey RK, Chao PC (2020) Optimizing a novel PPG sensor patch via optical simulations towards accurate heart rates. Microsyst Technol. https://doi.org/10.1007/s00542-020-04895-6

Sharma A, Polley A, Seung L, Sriram N, Wen TS, Srinath R (2017) A sub-60- $\mu$ A multimodal smart biosensing $\mathrm{SoC}$ with $>80-\mathrm{dB}$ SNR, $35-\mu \mathrm{A}$ photoplethysmography signal chain. IEEE J Solid-State Circuits. https://doi.org/10.1109/JSSC.2016.2642205

Sheng SS, Huang SC, Tran TH, Shao KY, Chao PCP, Chiang PY (2016) A $1.5 \mathrm{~mW}$ front-end readout circuit for a small-sized melanin sensor. Microsyst Technol 22(6):1449-1465

Shiue M, Yao K, Gong CA (2011) Tunable high resistance voltagecontrolled pseudo-resistor with wide input voltage swing capability. Electron Lett 47(6):377-378. https://doi.org/10.1049/el. 2010.3286

Shu Y-S et al. (2020) 26.1 A $4.5 \mathrm{~mm}_{2}$ multimodal biosensing SoC for PPG, ECG, BIOZ and GSR acquisition in consumer wearable devices. In: 2020 IEEE International Solid- State Circuits Conference - (ISSCC) (2020), pp 400-402

Sommermeyer D, Zou D, Ficker JH, Randerath W, Fischer C, Penzel T, Sanner B, Hedner J, Grote L (2016) Detection of cardiovascular risk from a photoplethysmographic signal using a matching pursuit algorithm. Med Biol Eng Comput 54(7):1111-1121

Tang Z, Tamura T, Sekine M, Huang M, Chen W, Yoshida M, Sakatani K, Kobayashi H, Kanaya S (2017) A chair-based unobtrusive Cuffless blood pressure monitoring system based on pulse arrival time. IEEE $\mathrm{J}$ Biomed Health Inform 21(5):1194-1205

Wong AK, Leung KN, Pun KP, Zhang YT (2010) A 0.5-Hz high-pass cutoff dual-loop transimpedance amplifier for wearable NIR sensing device. IEEE Trans Circuits Systems II Exp Briefs 57(7):531-535

Wu S, Shu Y, Chiou AY, Huang W, Chen Z, Hsieh H (2020) 9.1 A current-sensing front-end realized by a continuous-time incremental ADC with 12b SAR quantizer and reset-then-open resistive DAC achieving $140 \mathrm{~dB}$ DR and $8 \mathrm{ppm}$ INL at $4 \mathrm{kS} / \mathrm{s}$. In: 2020 IEEE International Solid- State Circuits Conference (ISSCC), San Francisco, CA, USA, 2020, pp 154-156, doi: https://doi.org/10.1109/ISSCC19947.2020.9062990

Yan et al. (2019) Novel deep convolutional neural network for cuffless blood pressure measurement using ECG and PPG signals. In: 2019 41st Annual International Conference of the IEEE Engineering in Medicine and Biology Society (EMBC), Berlin, Germany, 2019, pp. 1917-1920, doi: https://doi.org/10.1109/ EMBC.2019.8857108

Zheng Y, Yan BP, Zhangand Y-T, Poon CCY (2014) An armband wearable device for overnight and cuff-Less blood pressure measurement. IEEE Trans Biomed Eng, 61(7)

Publisher's Note Springer Nature remains neutral with regard to jurisdictional claims in published maps and institutional affiliations. 